\title{
Three-gradient regular solution model for simple liquids wetting complex surface topologies
}

\author{
Sabine Akerboom, Marleen Kamperman and Frans A. M. Leermakers*
}

\author{
Full Research Paper \\ Address: \\ Physical Chemistry and Soft Matter, Wageningen University, \\ Stippeneng 4, 6708 WE Wageningen, Netherlands \\ Email: \\ Frans A. M. Leermakers* - frans.leermakers@wur.nl \\ * Corresponding author \\ Keywords: \\ inverse opal; regular solution model; self-consistent field theory; \\ surface topology; wetting
}

Beilstein J. Nanotechnol. 2016, 7, 1377-1396.

doi:10.3762/bjnano.7.129

Received: 25 April 2016

Accepted: 25 August 2016

Published: 04 October 2016

This article is part of the Thematic Series "Biological and biomimetic materials and surfaces".

Guest Editor: S. N. Gorb

(C) 2016 Akerboom et al.; licensee Beilstein-Institut.

License and terms: see end of document.

\begin{abstract}
We use regular solution theory and implement a three-gradient model for a liquid/vapour system in contact with a complex surface topology to study the shape of a liquid drop in advancing and receding wetting scenarios. More specifically, we study droplets on an inverse opal: spherical cavities in a hexagonal pattern. In line with experimental data, we find that the surface may switch from hydrophilic (contact angle on a smooth surface $\theta_{Y}<90^{\circ}$ ) to hydrophobic (effective advancing contact angle $\theta>90^{\circ}$ ). Both the Wenzel wetting state, that is cavities under the liquid are filled, as well as the Cassie-Baxter wetting state, that is air entrapment in the cavities under the liquid, were observed using our approach, without a discontinuity in the water front shape or in the water advancing contact angle $\theta$. Therefore, air entrapment cannot be the main reason why the contact angle $\theta$ for an advancing water front varies. Rather, the contact line is pinned and curved due to the surface structures, inducing curvature perpendicular to the plane in which the contact angle $\theta$ is observed, and the contact line does not move in a continuous way, but via depinning transitions. The pinning is not limited to kinks in the surface with angles $\theta_{\text {kink }}$ smaller than the angle $\theta_{\mathrm{Y}}$. Even for $\theta_{\text {kink }}>\theta_{\mathrm{Y}}$, contact line pinning is found. Therefore, the full 3D-structure of the inverse opal, rather than a simple parameter such as the wetting state or $\theta_{\text {kink }}$, determines the final observed contact angle.
\end{abstract}

\section{Introduction}

Wetting of surfaces is a key feature for many applications. The wetting properties of a surface depend on both the material and the surface topography. A famous example is the surface of a lotus leaf: Although the material of the leaf is hydrophilic (contact angle on a smooth substrate $\theta_{\mathrm{Y}}<90^{\circ}$ ), the structured sur-

face is hydrophobic (apparent contact angle $\theta>90^{\circ}$ ) [1] Recently, different surface structures have been designed and fabricated from hydrophilic materials that show hydrophobic contact angles [2-10]. An example is an inverse opal as schematically shown in Figure 1. Our group recently reported 
an increase of $\theta$ from ca. $80^{\circ}$ to ca. $110^{\circ}$ for an inverse opal of polypyrrole [10].

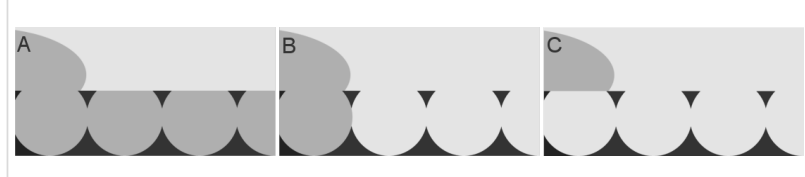

Figure 1: Schematic representation of the three wetting states on an inverse opal, A) impregnated state, B) Wenzel state, C) Cassie-Baxter state.

Our study is targeted to obtain (close to) molecular level insight in the wetting features of such surfaces using a simplistic modelling toolbox based on regular solution theory. To understand the increase in $\theta$, which is observed at macroscopic length scales, details about the microscopic scale should be considered. For the simplest case in which a water droplet wets the structured surface on a microscopic level with its preferred angle $\theta_{\mathrm{Y}}$ (see Figure 1B), the apparent contact angle, $\theta_{\mathrm{W}}$ is given by [11]

$$
\cos \theta_{\mathrm{W}}=r \cos \theta_{\mathrm{Y}}
$$

with $r$ the roughness of the surface (true contact area/projected area). This is called the Wenzel state, and it always magnifies the underlying wetting properties: $\theta$ decreases for hydrophilic materials and increases for hydrophobic materials. As the structured surfaces of interest, which are composed of a hydrophilic material, show an increase in $\theta$, this implies that the droplet in these systems cannot be in the Wenzel state, or that the assumption of this model, namely that the parameter $r$ captures all features of a surface topography relevant for the final droplet shape, is too simplistic.

A possible explanation of the increase in $\theta$ on structured surfaces, is air entrapment $[12,13]$. Air acts as hydrophobic patch $\left(\theta_{\mathrm{Y}}\right.$ for the water/air interface is $\left.180^{\circ}\right)$, and these patches lower the average surface energy of the surface (see Figure 1C) $[14,15]$. The resulting apparent contact angle for this so-called Cassie-Baxter state is then given by [16]

$$
\cos \theta_{\mathrm{CB}}=\Phi_{\mathrm{s}} \cos \theta_{\mathrm{Y}}-\left(1-\Phi_{\mathrm{s}}\right)
$$

with $\Phi_{\mathrm{S}}$ the fraction under the droplet that is in contact with the solid and $\left(1-\Phi_{\mathrm{S}}\right)$ the fraction under the droplet in contact with air. This approach thus defines the solid as a new material with a different effective surface energy on a macroscopic scale, and does not entail details about the droplet shape close to the surface structures on a microscopic level.
Another explanation of the difference in $\theta$ for a structured and unstructured surface of the same material is contact line pinning [17-20]. The three-phase contact line is hereby immobilized. Apart from chemical heterogeneities (which will not be discussed here), pinning occurs for a simple 1D system when the contact line encounters a kink in the surface, indicated with angle $\theta_{\text {kink }}$ in Figure 2. If $\theta_{Y}<\theta_{\text {kink }}$, the angle of the droplet with respect to the surface should exceed $\theta_{Y}$ in order to wet the surface after the kink (dotted area in Figure 2), and the droplet is thus pinned.

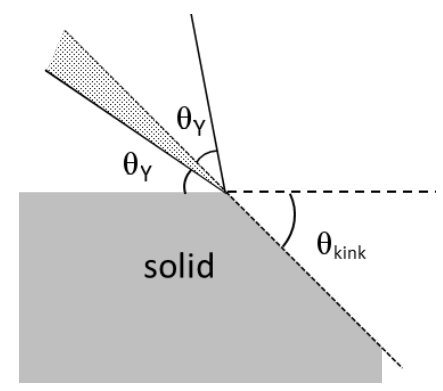

Figure 2: Surface structure induced contact line pinning in 1D: pinning occurs when $\theta_{Y}<\theta_{\text {kink }}$ (dotted area). The two $\theta_{Y}$ indicate the contact angle on the surface before and after the droplet has reached the kink.

However, pinning cannot result in any arbitrary shape. The mean curvature $J$ of the liquid/vapour $(\mathrm{L} / \mathrm{V})$ interface of a droplet, is related to the pressure difference across the $\mathrm{L} / \mathrm{V}$ interface, $\Delta P$, and the interfacial tension $\gamma$ according to the Young-Laplace equation [17]:

$$
\Delta P=2 J \gamma
$$

$\Delta P$ and $\gamma$ can be considered constant for a droplet (neglecting small curvature corrections, the deformation due to gravity and the near surface contributions expressed in the disjoining pressure), thus $J$ should also be constant [13]. This implies that if the surface structure induces a curvature in one direction due to pinning, this should be compensated by the opposite curvature in the perpendicular direction. Hence, if structuring of a surface induces a noticeable curvature of the droplet parallel to the surface, this should lead to deformations of the droplet perpendicular to the surface. As the latter curvature is coupled to the apparent contact angle, which is commonly measured perpendicular to the surface, we notice contact angle variations.

The feature of hydrophilic surfaces showing hydrophobic contact angles is, for reasons mentioned above, often linked to re-entrant angles of the surface structures [21,22]. An additional argument besides pinning, is that the liquid/air interfacial area 
should increase upon penetration of the liquid, creating more liquid/vapour interface [13]. This may imply that air entrapment occurs, even for hydrophilic materials $[12,23]$.

Since it is difficult to observe local curvatures in the three phase contact line experimentally $[18,24,25]$, in this study we turn to 'experiments in silico'. The present study is targeted to obtain insight in the wetting features of surfaces of hydrophilic materials that show hydrophobic contact angles and to differentiate between air entrapment and contact line pinning using a modelling approach.

Macroscopic approaches such as solving the Young-Laplace equation [26,27], minimizing the availability [28], or using geometry and energy [12] to find the droplet shape, do not take molecular details into account, and often require the contact angle as input parameter. Furthermore, air entrapment and coalescence [29] cannot be obtained by solving the Young-Laplace equation, and surfaces with re-entrant curvatures give impossible solutions [29]. Phase field methods [29], molecular dynamics (MD) [23,30-32], and mesoscopic lattice-Boltzmann (LB) models [33-37] are a viable option for this problem, but are challenging because wetting on complex structures involves multiple length scales [38] and the time needed to converge to a solution can be long $[31,38]$.

In this paper we focus on the very well known regular solution theory, which is frequently used throughout the field of physical chemistry, but not so often applied for studying wetting on complex surface topologies in three dimensions. Our models can be solved using a surprisingly simple algorithm (e.g., a Pikar iteration) on a desktop PC in a few minutes CPU time. However, similar to some of the theoretical approaches mentioned above, there are limitations with respect to the size of the systems that realistically can be considered. Albeit these limitations can easily be lifted by a factor of ten when the equations are solved using modern supercomputer facilities (which we here did not do). Here we focus on equilibrium and metastable states, which allows us to consider both advancing as well as receding contact angles. Even though the regular solution model is very well known, we will start by giving some backgrounds and highlights of the regular solution model. This gives us an opportunity to fix some of our parameters in the system. We then present the model and study the wetting of inverse opal structures.

\section{Results and Discussion Regular solution theory}

The start is a lattice model wherein the sites with linear length $b$ are arranged in a cubic lattice geometry, that is, each cell has $Z=6$ neighbours. Let there be $M$ sites in the system and thus the volume is given by $V=M b^{3}$. Sites are either filled by a solvent molecule, or the site is empty. The latter sites are said to be vacant and the number of vacant sites is $N_{\mathrm{V}}$. The remaining sites are filled by solvent and hence there are $N=M-N_{\mathrm{V}}$ sites filled. It is assumed that the solvent molecules only interact with each other when they occupy neighbouring sites and in this setting it is common to introduce the dimensionless Flory-Huggins interaction parameter, which is an Archimedean-like parameter needed for unlike contacts:

$$
\chi=\frac{Z}{2 k_{\mathrm{B}} T}\left(2 U_{\mathrm{LV}}-U_{\mathrm{LL}}-U_{\mathrm{VV}}\right) .
$$

A positive value means that LL contacts and VV 'contacts' are favoured over LV ones and this implies a tendency towards demixing. When we assume random mixing (mean-field approximation) we can evaluate the mixing interaction energy in the system by $U_{\text {mix }}=N \chi \varphi_{\mathrm{V}}$, where we ignored boundary effects and $\varphi_{\mathrm{V}}=N_{\mathrm{V}} / M$ is the volume fraction of vacancies. The entropy of mixing can be evaluated when we assume once more that the sites are randomly filled by solvent. The total number of ways to arrange the fluid and the vacancies is given by $\Omega=\left(\begin{array}{l}M \\ N\end{array}\right)$ and the mixing entropy is found by $S_{\text {mix }}=-k_{\mathrm{B}} \cdot \ln \Omega=$ $-k_{\mathrm{B}}\left(N \cdot \ln \varphi+N_{\mathrm{V}} \cdot \ln \varphi_{\mathrm{V}}\right)$ with $\varphi=N / M$ and $k_{\mathrm{B}}$ the Boltzmann constant. The free energy of mixing is given by $F_{\text {mix }}=U_{\text {mix }}-T \cdot S_{\text {mix }}$. Introducing the dimensionless free energy density $f=F_{\text {mix }} /\left(M b^{3} k_{\mathrm{B}} T\right)$ wherein the thermal energy $k_{\mathrm{B}} T$ and the volume $V$ are used to reduce the free energy, we obtain the well-known regular solution free energy density:

$$
f=\varphi \ln \varphi+\varphi_{\mathrm{V}} \ln \varphi_{\mathrm{V}}+\chi \varphi \varphi_{\mathrm{V}}
$$

with $\varphi+\varphi_{\mathrm{V}}=1$. The first two terms are negative and promote the mixing of the solvent and vapour. The last term drives the demixing. The critical conditions are found by setting the second and third derivatives of the free energy density (Equation 5) with respect to the volume fraction of liquid to zero. From such analysis it is found that there is a solubility gap as soon as $\chi>\chi^{\mathrm{cr}}=2$. By symmetry the critical density $\varphi^{\mathrm{cr}}=1 / 2$.

\section{Liquid/vapour interface}

Very famous is the extension of the regular solution theory to the description of the $\mathrm{L} / \mathrm{V}$ interface. In the footsteps of van der Waals [39] we like to find the density profile across a L/V interface $\varphi(z)$. Here $z$ is a (lattice) coordinate running perpendicular to the interface. We set $z=0$ at the interface and consider a lattice model with layer numbers $z=-M,-(M-1), \ldots,-1,0$, $1, \ldots, M-1, M$. The boundary layers $-M$ and $M$ are taken large 
enough so that the interface is not perturbed. We generalise Equation 5 and define a dimensionless free energy $F$ as follows

$$
F=\sum_{-M}^{M} \varphi(z) \ln \varphi(z)+\varphi_{\mathrm{V}}(z) \ln \varphi_{\mathrm{V}}(z)+\chi \varphi(z)\left\langle\varphi_{\mathrm{V}}(z)\right\rangle
$$

where it is understood that the mean-field approximation is now applied along lattice layers. The angular brackets in the last term indicate that in the interaction term "curvature" information is included, which is needed to evaluate the number of liquid-vacancy contacts in the presence of density gradients. In continuous language, we need to introduce

$$
\langle\varphi\rangle=\varphi+\frac{1}{6} \Delta \varphi
$$

in the interaction term, which on a lattice and in a one-gradient functional of Equation 6, translates to a local averaging operation:

$$
\langle\varphi(z)\rangle=\frac{1}{6}(\varphi(z-1)+4 \varphi(z)+\varphi(z+1)) .
$$

The target is to find the best volume fraction profiles that optimise the free energy $F$. Results are summarised in Figure 3.

Two volume fraction profiles are presented in Figure 3A, which were found numerically by minimizing Equation 6, for two values of $\chi$, not far from but above $\chi^{\mathrm{cr}}=2$. We have set the liquid phase at negative values of $z$, whereas the vapour is at positive $z$. The position of the interface is set at $z=0$ found by searching for $\varphi=0.5$ (in three-gradient results we will find the interface by the same criterion). The profiles follow very accurately the tanh dependence (see Figure 3A for numerical results). We note that for $\chi=2.2$ used below, these results deviate from the analytical predictions. Far from the interface the volume fraction profile levels off to the binodal values. The difference in volume fractions between the binodal values, here defined by $\Delta \varphi=\varphi(-M)-\varphi(M)$, is indicated in Figure 3A. The width $W$ of the interface is numerically found by intersection of the tangent line at $z=0$ with the binodal value. We can evaluate the surface tension $\gamma$, which is given in units $k_{\mathrm{B}} T / b^{2}$, numerically, as discussed in section S1 of Supporting Information File 1.

In Figure 3B we prove that near the critical point (i) the surface tension, (ii) the width of the interface and (iii) $\Delta \varphi$ as found by our numerical solution accurately obey scaling relations with respect to the difference to the critical point $\Delta \chi=\chi-2$.

Interestingly, near the critical point there is an analytical route to optimise the free energy $F$ [40]. In short, near the critical point the density of the liquid (and thus also for the vacancies) is never far from the critical value. Introducing an order parameter $\varphi=\varphi-0.5$, we can write $F$ as a function of the order parameter and then Taylor series expand the logarithms up to the fourth order in the order parameter. As a result we obtain a Landau free energy in terms of the order parameter. An Euler-Lagrange optimisation then leads to the famous tanhprofile already known by van der Waals. We do not go into these details and mention that fully in line with the numerical results presented in Figure 3B the scaling exponents as found by this analytical route are in line with the numerical results: For the surface tension the (mean field) value is $-3 / 2$, it is $1 / 2$ for the width of the interface, while the difference in densities of the two phases vanishes with an exponent $-1 / 2$ [40].
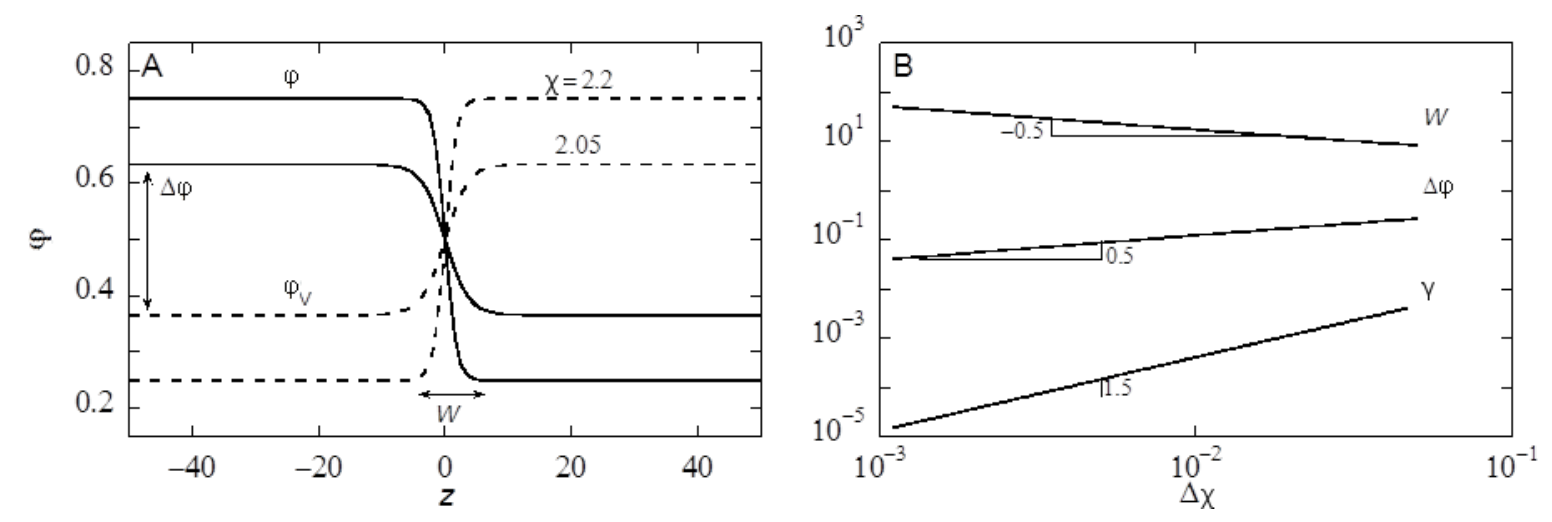

Figure 3: A) Examples of volume fraction profiles across a liquid/vapour interface found numerically by exact minimisation of Equation 6 , with the interaction parameter $X=2.05$ and $X=2.2$ as indicated. B) Width of the interface $(W)$, density difference between the two phases $(\Delta \varphi)$ and dimensionless surface tension $(\mathrm{Y}$ ) as function of $\Delta \mathrm{X}=\mathrm{X}-2$ in double logarithmic coordinates. 
Our aim is to present results that are relevant for the water/ vapour system. Of course a symmetric lattice model falls short in this respect, because it assumes that as much water will be in the vapour phase as free volume will be in the water phase. The symmetry can only be broken in a more elaborate model wherein water is more realistically represented. We mention that such an approach is (at least in principle) possible, but here we choose not to go into such complications. We know that at ambient temperatures the water/vapour system is not near critical. Indeed it is very far from critical. Hence it is necessary to choose a sufficiently high $\chi$ value. In a lattice model it is advised to keep the width of the interface larger than the size of a lattice site (i.e., $W>b$ ). In the other limit one experiences many so-called lattice artefacts, which may frustrate the analysis of the shape of a droplet on top of a structurally complex surface. For this reason we choose here $\chi=2.2$ for the liquid/ vapour interactions, unless stated otherwise. For this value the width of the interface $W$ is approximately 8 in units $b$. As the width of an air-with-water interface is just a few angstroms [41], we may infer that when the water/vapour system is the target of our calculations, the corresponding value of $b$ is a value less than an angstrom. Again we accept deviations from the air/water system and advice to consider the value of $b$ to be in the order of a few angstroms (say $0.2 \mathrm{~nm}$ ). The fraction of liquid in the liquid-rich phase for $\chi=2.2$ is about 0.7515 , and the fraction of liquid in the vapour-rich bulk phase has the binodal value $\varphi^{\#}=0.2485$. Again these values differ dramatically from our experimental system of water in air at $100 \%$ relative humidity. Finally, the interfacial tension in this system is given by $\gamma=0.03326$ in units $k_{\mathrm{B}} T / b^{2}$, which translates with $b=0.2 \mathrm{~nm}$ to $3.422 \mathrm{mN} / \mathrm{m}$. This value is smaller than the known value for water. All these differences with respect to our experimental system are accepted as we search only for scenarios. For ease of reference we may call the liquid-rich phase "water" and the vacancy-rich phase "vapour".

\section{Droplet on an unstructured solid}

Still using the one-gradient approach, it is possible to study wetting phenomena using the regular solution model. We need remarkably few modifications in the system. The only issue is that we need to introduce a substrate. To do so, we first specify the lattice coordinates $z=0,1,2, \ldots, M$, and introduce a surface component $\mathrm{S}$ as a boundary condition, that is, we choose $\varphi_{S}(0)=1$ and $\varphi_{S}(z)=0$ for all $z>0$. The liquid and the vapour are allowed to be in the half-space $z>0$. We have in principle two new interaction parameters $\chi_{\mathrm{LS}}$ and $\chi_{\mathrm{VS}}$ by introducing a "third" component.

Without losing generality we can set $\chi_{\mathrm{VS}}=0$, and keep $\chi_{\mathrm{S}}=\chi_{\mathrm{LS}}$ to specify the preferential adsorption of the liquid component on the surface. A negative value means that the solvent has a preference to sit next to the surface over the vapour. At $\chi_{\mathrm{S}}=0$ we expect a contact angle of $90^{\circ}$. Hydrophobic surfaces are modelled when $\chi_{S}>0$. We will mostly restrict ourselves to hydrophilic surfaces, thus to $\chi_{\mathrm{S}}<0$.

In the case of a L/V system next to a surface the regular solution free energy assumes the form

$$
\begin{array}{r}
F=\sum_{z=1}^{M} \varphi(z) \ln \varphi(z)+\varphi_{\mathrm{V}}(z) \ln \varphi_{\mathrm{V}}(z) \\
+\chi \varphi(z)\left\langle\varphi_{\mathrm{V}}(z)\right\rangle+\chi_{\mathrm{S}} \varphi(z)\left\langle\varphi_{\mathrm{S}}(z)\right\rangle,
\end{array}
$$

where it is understood that the last term is only non-zero when $z=1$, where it assumes the value $\chi_{S} \varphi(1)\left\langle\varphi_{S}(1)\right\rangle=\chi_{S} \varphi(1) / 6$.

There are several routes to study wetting. Our preference goes to study so-called adsorption isotherms. Of course we need a solubility gap and thus $\chi>2$ (we use a value of 2.2 throughout). Next, we consider a specific value of $\chi_{\mathrm{S}}<0$ and specify a given amount of solvent $\Gamma=N=\sum_{z=1}^{M} \varphi(z)$ in the system. We solve the self-consistent field equations and obtain the optimised density profile $\varphi(z)$. Far from the surface, the density profile converges to the bulk value $\varphi^{\mathrm{b}}$. The adsorbed amount (surface excess) of liquid is found by:

$$
\Gamma^{\sigma}=\sum_{z=1}^{M}\left(\varphi(z)-\varphi^{\mathrm{b}}\right)
$$

We focus on how the adsorption isotherms, $\Gamma^{\sigma}\left(\varphi^{b}\right)$, behave near the bulk binodal $\varphi^{\#}$. When upon the approach of the bulk binodal the adsorbed amount simply increases and diverges at the binodal value, we have a complete wetting situation and the contact angle is zero. Alternatively, the isotherm crosses the binodal at a finite value of the adsorbed amount, that is, for an amount $\Gamma^{\#}=\Gamma^{\sigma}\left(\varphi^{b}\right)<\infty$. We refer to this first crossing as the "microscopically thin film" adsorbed at the surface S. By means of a van der Waals loop the isotherm then returns to the binodal and approaches the infinite adsorbed amount upon the final approach towards the bulk binodal. We refer to this adsorbed amount as the "macroscopically thick film" on the surface. Such situation is typical for partial wetting states, where the macroscopically thick film represents the situation under a drop, and the thin film is found far away from the drop where a gas-like film resides on the substrate. As for each solution along the isotherm we have the surface tension accurately available from the self-consistent field (SCF) solution, we can find the contact angle from Young's law: 


$$
\cos \theta_{\mathrm{Y}}-1=\frac{\gamma_{\mathrm{SV}}-\left(\gamma_{\mathrm{SL}}+\gamma_{\mathrm{LV}}\right)}{\gamma_{\mathrm{LV}}}=\frac{\gamma_{\text {thin }}-\gamma_{\text {thick }}}{\gamma_{\mathrm{LV}}}
$$

where all interfacial tensions are computed for systems in which the chemical potential is that corresponding to the bulk binodal The value of $\gamma_{\text {thin }}$ is found from the first crossing of the binodal, and $\gamma_{\text {thick }}$ is the surface free energy in the system when there is a very thick adsorbed layer at the surface. Hence, we can obtain contact angle information without explicitly generating droplets. In passing we mention that for not too small droplets the contact angle as obtained by a three-gradient analysis (as used below) gives identical contact angles as the ones that follow from Equation 11, which used information from onegradient regular solution models.

The contact angle of a liquid droplet $\theta_{\mathrm{Y}}$ is calculated for various adsorption strengths $\chi_{\mathrm{S}}$ and for different strengths of interaction between liquid and vapour $\chi$ (Figure 4 ). The more negative $\chi_{S}$, the more favourable the interaction between $\mathrm{S}$ and $\mathrm{L}$, and the more the droplet spreads, resulting in smaller $\theta_{Y}$. Eventually, the liquid prefers to wet the solid completely, i.e., $\theta_{\mathrm{Y}}=0^{\circ}$. At $\chi$ near the critical value of 2 , the droplet enters the complete wetting regime $\left(\theta_{Y}=0^{\circ}\right)$ already for very low values of the surface affinity $\chi_{S}$. For strong segregations the interfacial energies increase and we need larger adsorption energies to enforce wetting.

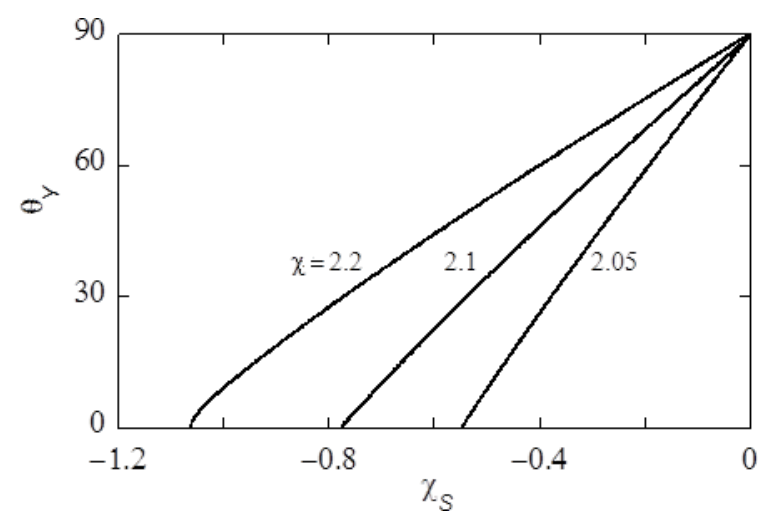

Figure 4: Contact angle of liquid on flat solid, $\theta_{Y}$ as function of the interaction parameter of the liquid with the solid, $Y_{S}$, for three different values of the interaction parameter of the liquid with the vapour, $Y$

In this paper we aim to mimic a polypyrrole surface for which the water contact angle of a smooth surface is about $\theta_{\mathrm{Y}}=80^{\circ}$. As we already selected $\chi=2.2$, we will be in the correct contact angle regime when we set the adsorption energies around $\chi_{\mathrm{S}}=-0.2$. Below we will always mention the strength of adsorption.

\section{Liquid condensation in parallel slit}

The surface structure of an inverse opal consists of close to spherical cavities. In such structures we should anticipate the occurrence of capillary condensation or, alternatively, capillary drying. For this reason we use the regular solution model to study classical capillary condensation. To this end we consider a system that contains two surfaces. One at $z=0$ and another one at $z=D+1$. Hence $\varphi_{\mathrm{S}}(z)=0$ for $z=1,2, \ldots, D$ and unity elsewhere. Now our regular solution free energy is given by

$$
\begin{aligned}
F= & \sum_{z=1}^{D} \varphi(z) \ln \varphi(z)+\varphi_{\mathrm{V}}(z) \ln \varphi_{\mathrm{V}}(z) \\
& +\chi \varphi(z)\left\langle\varphi_{\mathrm{V}}(z)\right\rangle+\chi_{\mathrm{S}} \varphi(z)\left\langle\varphi_{\mathrm{S}}(z)\right\rangle .
\end{aligned}
$$

In this case the last term automatically accounts for the interactions with the surfaces, as it is non-zero for $z=1$ and $z=D$. More specifically, $F$ has two surface contributions $\chi_{\mathrm{S}} \varphi(1) / 6+\chi_{\mathrm{S}} \varphi(D) / 6$.

As there are two interfaces, we anticipate the adsorption of the liquid onto both surfaces simultaneously. We want to record the adsorbed amount in the slit as a function of the (dimensionless) chemical potential $\left(\mu=\ln \varphi^{b}\right)$ of the liquid component. One complication arises because in layers $z=1, \ldots, D$, the bulk volume fraction may not be reached and we cannot simply "pick up" this value from the profiles. As explained in section S1 of Supporting Information File 1, the SCF protocol gives (as output) the volume fraction of a reference system that is in equilibrium with the molecules in the slit. This reference value is used to compute the isotherms. In Figure 5A we present an example for a slit distance $D=10$ (in lattice units) and our default interaction parameters $\chi=2.2$ and $\chi_{\mathrm{S}}=-0.3$. Recall that under these conditions the surfaces are preferentially solvated by the liquid, and $\theta_{\mathrm{Y}}$ is $68^{\circ}$. In such situations there is a large van der Waals loop in the adsorption isotherm (cf. Figure 5A). The Maxwell construction can be used to find where, in equilibrium, the step in the isotherm should take place. In line with the hydrophilic character of the surfaces we find the step in the subsaturated region. In Figure 5A the grey vertical line represents the bulk binodal value. The step takes place at a lower chemical potential (local binodal) than that corresponding to the bulk binodal. We define $\Delta \mu$ as the difference in $\ln \varphi^{\mathrm{b}}$ between the local and bulk binodals as indicated in Figure 5A.

It is important to consider the isotherm in slightly more detail. After the jump in the isotherm the adsorbed amount only marginally increases further: the isotherm continues into the supersaturated region and as the system increases the bulk volume fraction, in the limit of very high concentrations the 

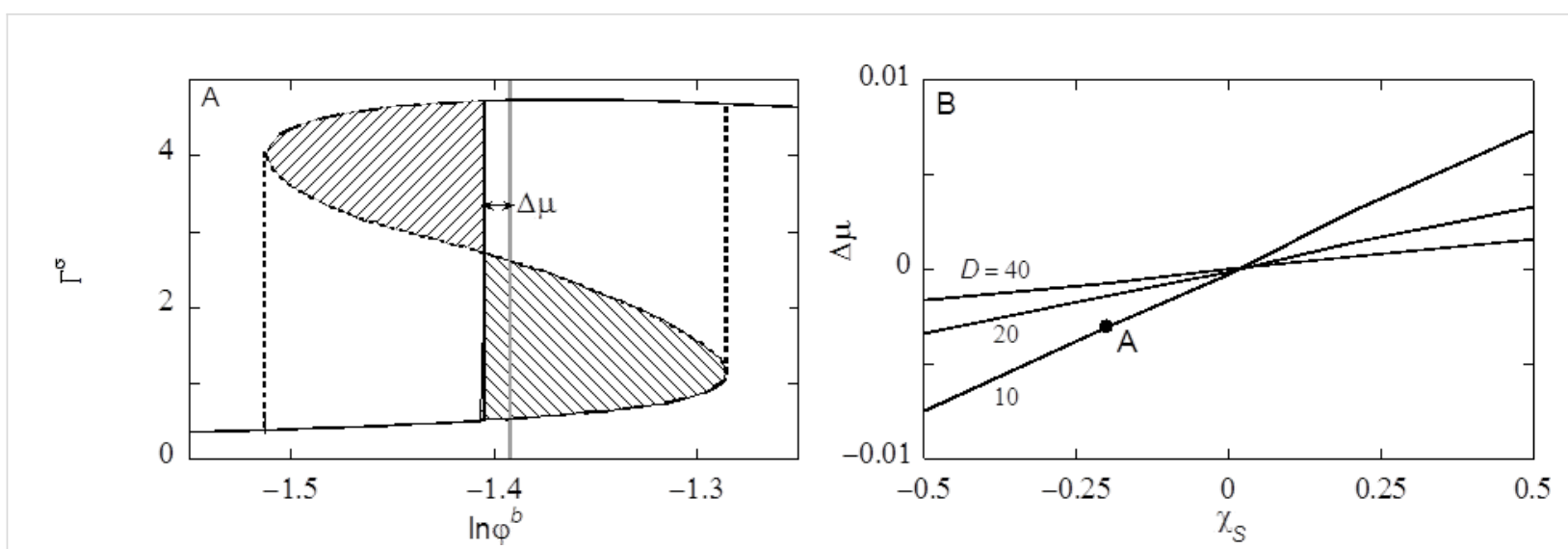

Figure 5: A) Adsorption amount $\Gamma^{\sigma}$ of the liquid component as a function of the volume fraction of liquid in the vapour $\varphi^{b}$ in lin-In coordinates $(x$-axis is chemical potential), in a slit with surfaces that are a distance $D=10$ apart and have an adsorption energy Xs $=-0.2$. The metastable branches are dashed. The unstable part is dotted. The chemical potential $\mu^{\#}$ is indicated as the grey vertical line. The solid vertical line is placed at the chemical potential where a step in the isotherm takes place (local binodal). This step is found by the Maxwell construction, that is by the equal area argument (the two shaded regions have the same area). The difference in chemical potential $\Delta \mu$ between the place of the step and the binodal is indicated. The vertical dotted lines are placed at chemical potentials corresponding to the spinodal points. B) The chemical potential where the step in the isotherm (compared to value of the bulk binodal) $\Delta \mu$ takes place (at equilibrium) as function of $X_{S}$ for slit distances $D=10,20$ and 40 as indicated. The solvent-vacancy interaction is set to $X=2.2$. The label $A$ on the curve for $D=10$ corresponds to the condition used in panel $A$.

excess should go down again to become zero in the limit of $\varphi^{\mathrm{b}}$ to 1 .

The most interesting feature of the isotherm is the presence of a loop. Associated to the loop there are two spinodal points. These spinodal points are located at the turning points of the isotherm. There are two regions of metastability, namely between the local binodal and the first turning point. This spinodal point is found in the region of supersaturation. The other metastable region is found between the turning point in the subsaturated region and the local binodal in the top region of the isotherm. In the isotherm the metastable branches are indicated by dashed line parts. When in the absence of strong fluctuations the bulk volume fraction is increased, the slit may not necessarily change its contents at the local binodal, but instead remains dry up to - in the extreme case - the spinodal point is reached, and the slit is filled with liquid following the dashed line. Inversely, when the slit is wet, and the bulk concentration is reduced, the drying does not necessarily take place at the local binodal, but enter the other metastable branch. Again the drying must take place before or at the lower spinodal point (following the other dashed line). Hence in dynamical situations a hysteresis loop may be followed where the steps at the spinodal are indicated by the vertical dotted lines. The spinodal points have important roles in the advancing or receding contact line calculations (see below). Even though the van der Waals loop in the isotherm is due to the mean-field approximation, it is found that in real life experiments the system also may be trapped in metastable states very much alike those found in the mean-field model.
In Figure 5B we report that the difference between the local and the bulk binodal in confined spaces is a function of the affinity of the solvent for the substrate. When $\chi_{S}$ is more negative the $\Delta \mu$ increases to more negative values. Indeed when $\chi_{S}>0$, that is for hydrophobic surfaces, the local binodal occurs at supersaturated solutions. With increasing $D$ the local binodal shifts towards the bulk binodal.

\section{Curved L/V interfaces: Kelvin and Laplace}

Macroscopic droplets (with negligible curvature) cannot be generated using our method. As the system size is limited, our drops have L/V interfaces that are typically strongly curved. The thermodynamics of curved interfaces is well understood, but there are several complications. One of the issues is that the location of the interface is somewhat arbitrary. On top of this the interfacial tension in curved interfaces cannot uniquely be computed. It depends on the notion of the position of the interface. There exists a choice of the position of the interface, the so-called surface of tension, for which a small notional change of the radius does not influence the value of the surface tension. For this special case the Laplace equation simplifies to Equation 3, and the value of the interfacial tension does not deviate much from the planar value.

From the Laplace equation we know that in droplets with curved $\mathrm{L} / \mathrm{V}$ interfaces there is a Laplace pressure. As a consequence the chemical potential of the liquid in a drop is at a higher chemical potential compared to systems with planar interfaces. The increased chemical potential is reflected in the oversaturation of water in the vapour phase; a phenomenon 
named after Kelvin. With oversaturation of the system, which necessarily occurs in our calculations due to the finite size of our droplets, one will invariably get closer to the spinodal point of the capillary condensation process. Hence, oversaturation may trigger the filling of confined regions by the liquid. Small droplets cause a stronger oversaturation than larger droplets and the presence of small droplets may result in a spontaneous filling of the voids by capillary condensation when this may not yet occur for larger drops.

The radius specified by the surface of tension $R_{\mathrm{SOT}}$ coincides with the visual inspection of where the interface is for many systems. Below we therefore do not exactly determine the exact $R_{\mathrm{SOT}}$ and use the Ansatz that the interface position is where the solvent volume fraction hits the value $\varphi=0.5$.

\section{Three-gradient regular solution model}

Let us next extend the regular solution theory to model liquid drops at a complex surface topology. We consider a threegradient coordinate system $\mathbf{r}=(x, y, z)$ with $x=1,2, \ldots, M_{x}$, $y=1,2, \ldots, M_{y}$ and $z=1,2, \ldots, M_{z}$. In contrast to the onegradient systems where the surfaces were treated through the boundary conditions, in three gradient models it is more natural that the surface component $\mathrm{S}$ will occupy lattice sites within the specified volume. Hence, we will specify all the lattice sites within the system: the volume fraction of $S$ is unity and the remainder of the lattice sites are filled by the liquid and vapour components in the usual way. The regular solution free energy is straightforwardly generalised and both interactions between $\mathrm{L}$ and $\mathrm{V}$ as well as with the surface component $\mathrm{S}$ are accounted for:

$$
\begin{gathered}
F=\sum_{x=1}^{M_{x}} \sum_{y=1}^{M_{y} M_{z=1}} \sum_{z}(\mathbf{r}) \ln \varphi(\mathbf{r})+\varphi_{\mathrm{V}}(\mathbf{r}) \ln \varphi_{\mathrm{V}}(\mathbf{r}) \\
+\chi \varphi(\mathbf{r})\left\langle\varphi_{\mathrm{V}}(\mathbf{r})\right\rangle+\chi_{\mathrm{S}} \varphi(\mathbf{r})\left\langle\varphi_{\mathrm{S}}(\mathbf{r})\right\rangle
\end{gathered}
$$

wherein the angular brackets indicate that the free energy accounts for the "curvature" information in three directions. The lattice implementation is simply:

$$
\begin{aligned}
\langle\varphi(\mathbf{r})\rangle= & \langle\varphi(x, y, z)\rangle \\
= & \frac{1}{6}(\varphi(x-1, y, z)+\varphi(x+1, y, z) \\
& +\varphi(x, y-1, z)+\varphi(x, y+1, z) \\
& +\varphi(x, y, z-1)+\varphi(x, y, z+1)) .
\end{aligned}
$$

Mirror-like, no-gradient boundary conditions are implemented in boundary layers in the system. This is implemented by setting $\varphi(0, y, z)=\varphi(1, y, z), \varphi\left(M_{x}+1, y, z\right)=\varphi\left(M_{x}, y, z\right)$, and similarly for the other boundaries in $y$ - and $z$-directions. Using these boundary conditions it is possible to consider a representative part of the surface, while keeping the computation times and system volumes to a minimum.

In the calculations there are no assumptions regarding the effects of the line tension. The model fully accounts for these effects, but the line tension contributions in our systems were not explicitly extracted. The most important reason why we did not do so is that the line tension cannot be uniquely extracted from the overall grand potential, because a choice for the position of the three-phase contact line is required.

\section{Specifying the inverse opal}

The regular solution free energy of Equation 13 still requires detailed information on the distribution of the solid material $\mathrm{S}$ in the inverse opal. The idea is to consider a representative piece of a substrate that contains spherical cavities in a specified arrangement (i.e., crystalline ordering with close to hexagonal or square packing symmetries). The cut-off height controls the opening of the cavities as shown in the example of Figure 6. The parameters that control the surface topology are listed in Table 1. Below we will use the parameter $c=h / d$, which is a fraction at which the cavities were cut and $\Phi_{\mathrm{S}}$ is the fraction of the "top" of the surface that is solid.

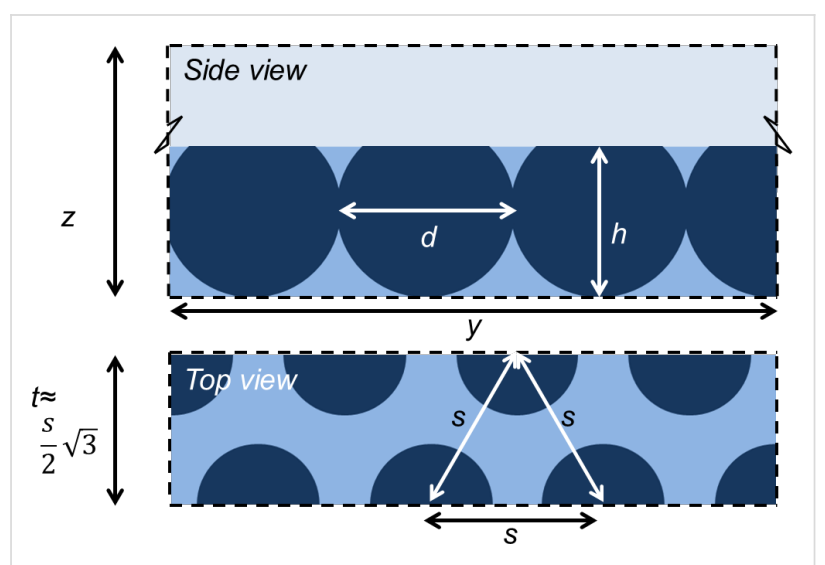

Figure 6: Schematic side view $(z, y)$-plane and top view $(x, y)$-plane of an inverse opal with two rows of $n=3.5$ cavities in a staggered packing ( 3.5 cavity volumes are in the system, four cavity positions are required). The distance between two $(m=2)$ rows is $t$. For a hexagonal packing the two rows are displaced with respect to each other by a value $t \approx(s / 2) \sqrt{3}$, where $s$ is the distance between the cavities. On a lattice $t$ must be an integer. Reflecting boundaries are applied in all directions.

The parameters of Table 1 completely specify how many cavities are present in the computation volume. Let the cavities be 
Table 1: Parameters that determine the structure of the inverse opal. Even and odd rows of cavities are displaced by half the distance between the cavities in a row, that is, by $s / 2$. All quantities are given in lattice units, that is in values of $b$. Below also the cut-off ratio $c=h / d$ (to specify which part of the cavity is cut-off) and $\Phi_{S}$ (the fraction of the top of the surface that is solid) is used.

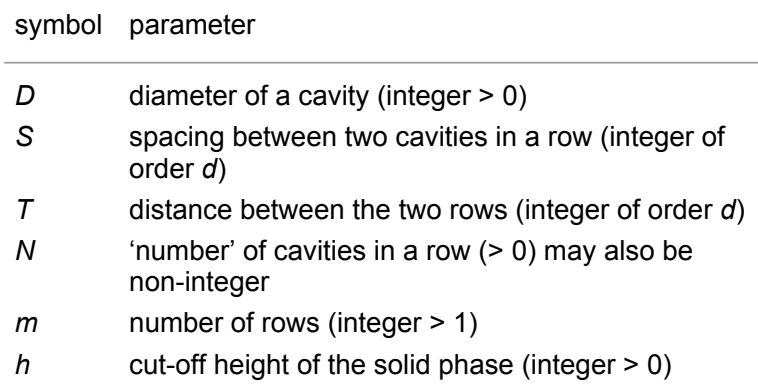

numbered by $i=1,2,3, \ldots, N_{\mathrm{c}}$. In the example of Figure 6 there are two rows of 3.5 cavities and thus we need $i=1, \ldots, 8$ cavity positions. The input parameters thus specify the coordinates of the cavities $\left\{\mathbf{r}_{1}, \mathbf{r}_{2}, \ldots, \mathbf{r}_{i}, \ldots, \mathbf{r}_{N \mathrm{c}}\right\}$. The cavities in the inverse opal are placed in rows along the $y$-direction, the direction perpendicular to the rows is the $x$-direction and the cavities are positioned at the lowest $z$-values possible, that is for all cavities $i, r_{i z}=d / 2$ (cavity radius).

The solid phase extends up to a height $z=h$ where $h$ is the cut-off height. The solution above the inverse opal starts at a height $z>h$. The first and last row in the $y$-direction have their centres on the boundary that is at $y=1 / 2$ and $y=M_{y}+1 / 2$, respectively. This implies that the system size in the $y$-direction is $M_{y}=(\mathrm{m}-1) t$, where $t$ is the distance between two rows and $m$ is the number of rows. The system size in the $x$-direction is given by $M_{x}=n s$, with $n$ the number of cavities in a row (when half the cavity is in the system the cavity counts by 0.5 ), and $s$ the distance between cavities in a row. In the example of Figure $6, M_{x}=3.5 \mathrm{~s}$. The system size in the $z$-direction should exceed $h$ sufficiently so that a sessile drop can be on the substrate. In the first row the first cavity is by default with its centre at $x=1 / 2$, that is, at the lower boundary. The first cavity in the second row is positioned at $x=(s+1) / 2$, et cetera.

All non-zero surface densities can now be computed. When for a coordinate $\mathbf{r}^{\prime}$ the distance to all of the coordinates $\left\{\mathbf{r}_{1}\right.$, $\left.\mathbf{r}_{2}, \ldots, \mathbf{r}_{i}, \ldots, \mathbf{r}_{N \mathrm{c}}\right\}$ is larger than the radius of a cavity, i.e., $d / 2$ and when the $z$-value is less or equal to $h$, we set $\varphi_{S}\left(\mathbf{r}^{\prime}\right)=1$ and $\varphi_{\mathrm{S}}\left(\mathbf{r}^{\prime}\right)=0$, otherwise:

$$
\varphi_{\mathrm{S}}\left(\boldsymbol{r}^{\prime}\right)= \begin{cases}1 & \mathbf{r}_{z} \leq h \text { and } \quad\left|\mathbf{r}_{i}-\mathbf{r}^{\prime}\right|>\frac{d}{2} \quad \forall i \\ 0 & \text { otherwise }\end{cases}
$$

This solid distribution is fixed during the free energy optimisation. Of course only the coordinates that are not taken up by $\mathrm{S}$ can be filled with L or V. On the "top" of the solid phase it is of interest to know the fraction of sites occupied by S. These are easily evaluated by

$$
\Phi_{\mathrm{S}}=\frac{1}{M_{x} M_{y}} \sum_{x=1}^{M_{x}} \sum_{y=1}^{M_{y}} \varphi_{\mathrm{S}}(x, y, h) .
$$

Analytical estimates of Equation 16 are given in section S2 of Supporting Information File 1. The cut-off height $h$ normalised by the particle diameter $d$ will be referred to by $c$ :

$$
c=\frac{h}{d} \text {. }
$$

Below we will be interested in hexagonally ordered cavities. For an optimal hexagonal packing, the distance $s$ between the particles along a row and the distance $t$ between the rows, should obey $t=(s / 2) \sqrt{3}$. However, on the lattice only integer values are allowed. Rounding to closest integer values must be implemented. For some values of the particle distances $s$ there is a reasonable value of $t$, for other distances the error is relatively large. Only values of $s$ which require rounding errors below 0.15 for the corresponding $t$ value are used.

The parameters in Table 1 can be used to generate a large variety of inverse opal structures. As long as $s>d$, we have the situation that the cavities are isolated. However, the cavities may become interconnected when $s<d$. In experimental situations such overlap of cavities may occur, and then there are usually small openings connecting the cavities. This is why this particular parameter setting is allowed.

\section{Example 1: liquid condensation in a weakly hydrophilic face centred square inverse opal}

We first consider a simple inverse opal structure that has cavities with a diameter $d=31$. The distance $s$ between the cavities is set to 88 , and the distance between the rows $t=44$. This implies a face-centred square arrangement of the cavities. The number of cavities in a row is $n=1$, whereas the number of rows is set to 3 . As can be seen from Figure 7, this setting generates an equal box size in $x$ - and $y$-directions. As the distance between the cavities exceeds the cavity diameter, we have isolated pockets. The surface interaction is set to a slightly hydrophilic value $\chi_{\mathrm{S}}=-0.3$.

To compute the adsorption isotherm we start with a low amount of liquid in the system and then increase this amount step by 

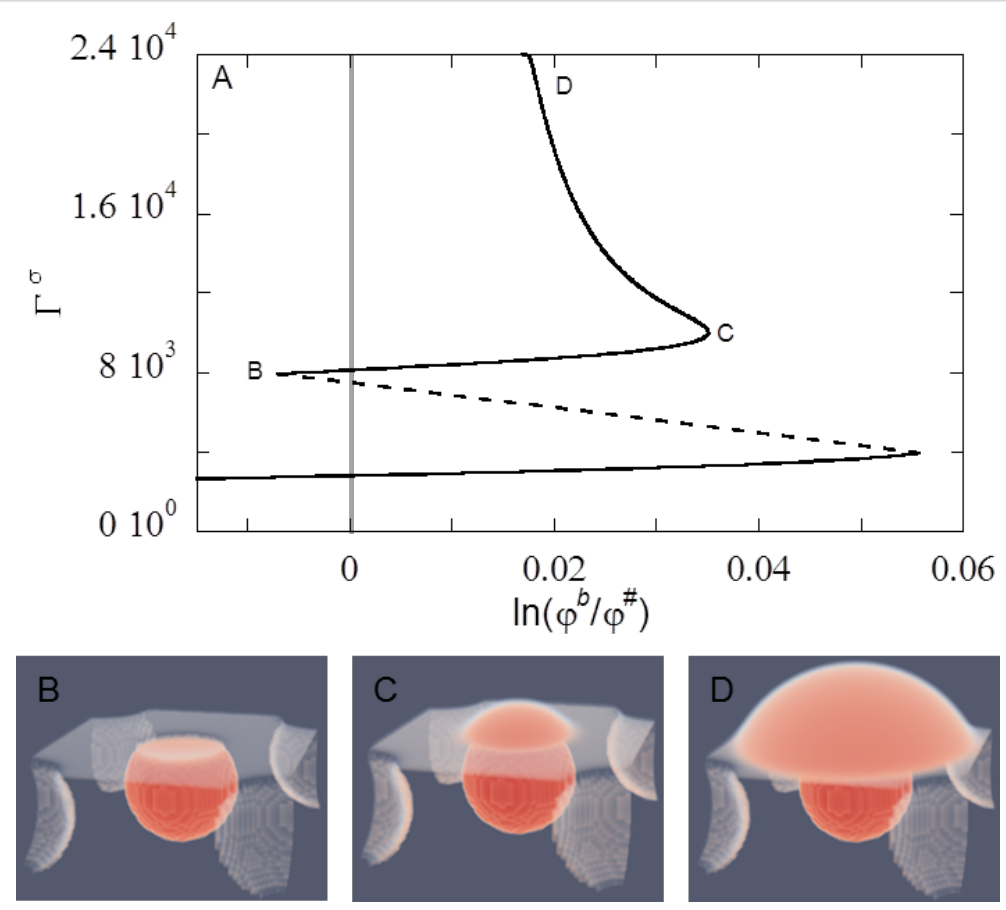

Figure 7: Liquid condensation in hydrophilic inverse opal $(d=31, s=88, t=44, m=3, n=1, h=25(c=0.8))$. A) Excess adsorbed amount of the liquid component in the system as a function of the volume fraction of the liquid normalised by the binodal value in lin-In coordinates. The dashed part represents not the true part of the isotherm but rather connects two states, before the condensation of a pocket and after the condensation. The dotted, vertical line is at the bulk binodal $\varphi^{\#}$. The labels along the isotherm refer to the snapshot colour-coded density distributions given in panels B, C, D. Red is the high-density liquid phase, white is the low-density (gas) phase.

step. In the calculations the outcome of a given calculation serves as an initial guess for the subsequent calculation. This is why a system can be trapped in metastable states, similar to the experimental counterparts.

In Figure 7A we present the adsorbed amount of the liquid component $\Gamma^{\sigma}=\Gamma-V^{\prime} \varphi^{\mathrm{b}}$, where $V^{\prime}$ is the volume available for the $\mathrm{L}$ and $\mathrm{V}$ components, which is $V^{\prime}=M_{x} M_{y} M_{z}-\Sigma_{\mathrm{r}} \varphi_{\mathrm{S}}(\mathbf{r})$, as a function of the volume fraction of the liquid component in the bulk. Here we did not normalise with respect to the available surface area and thus this amount is proportional to the surface area. The bulk volume fraction is normalised by the bulk binodal value. The curve is plotted in lin-ln coordinates. Upon an increase in the amount of liquid component in the system $\Gamma$, first the vapour phase is gradually saturated with liquid and the adsorbed excess remains modest: At the surface a gaseous adsorption layer develops. Then, upon further increase of the amount, the binodal value is crossed and the system enters the super-saturation regime. The liquid film remains homogeneous along the surface, until $\Gamma^{\sigma} \approx 4000$, then a first-order jump in the isotherm takes place (cf. Figure 7A, dotted line). As can be seen in Figure $7 \mathrm{~B}$, at this stage a droplet formed in the confined space of the cavity and the curvature on the L/V interface in the opening is concave, resulting in a negative Laplace pressure inside the droplet. Since the chemical potential of the liquid molecules should be the same everywhere, this means that the vapour phase is under-saturated (see Figure 7A).

The volume fraction of liquid in the bulk $\varphi^{\mathrm{b}}$ increases as more liquid is added and passes the binodal value $\varphi^{\#}$ again. At this binodal point there is no under- or oversaturation, hence no curvature of the $\mathrm{L} / \mathrm{V}$ interface at the opening (not shown). Additional liquid that is added to the droplet induces a convex curvature on top of the droplet, and the bulk oversaturates up to $\mathrm{C}$ in Figure 7A. The value of $\Gamma^{\sigma}$ at this point is approximately 10000 , and consists of a thin film $\left(\Gamma^{\sigma} \approx 4000\right)$ and the macroscopic droplet $\left(\Gamma^{\sigma} \approx 6000\right)$. The volume of a sphere with $d=31$ is about 15600 . Given that the density difference between the liquid-rich and vapour-rich phase is about 0.5 , and that part of the thin liquid film becomes part of the macroscopic droplet, the valur of $\Gamma^{\sigma}$ found at point $C$ is in agreement with what is expected for a cavity with diameter $d=31$.

Additional liquid is subsequently used not to fill the other cavities, but to increase the volume of the existing droplet (Figure 7D) and the droplet starts to spread on the substrate. Below we will follow this process in a slightly different geometry. The oversaturation needed for capillary condensation to occur for the other cavities is in this case not reached. This means that for this inverse opal, with $d=31$, once condensation 
has taken place, a droplet grows on top of the filled cavity and the remaining cavities are not filled via capillary condensation, but typically rather fill once the central droplet spreads over and on top of the other cavities (not shown).

Most inverse opals in experiments, which are fabricated using sacrificial particles, have cavities of hundreds of nanometres or more $[3,10,42-44]$. These sizes are much larger than the cavities considered in the current calculations. Smaller cavities can fill more easily via capillary condensation (cf. Figure 5B). Since the impregnating wetting state is not observed even for such small cavity sizes as used in Figure 7, we conclude that the impregnating wetting state due to capillary condensation is not likely to develop for practical inverse opals which are marginally hydrophilic.

In this surface structure, the cavities are not connected, whereas for some experimentally fabricated inverse opals all cavities may be interconnected via a small opening. Since the curvature of the droplet should be constant, the curvature of the liquid-vapour interface at the small opening is the same as for the rest of the macroscopic droplet [13], and hence the high curvature needed for the next cavity to be wetted via this opening, is not reached. Therefore, a cavity that is filled with liquid will wet the next cavity via the larger opening at the top, rather than through this small hole (not shown).

\section{Droplets on top of the hexagonally ordered inverse opal}

In the remainder of this paper we will focus on close-to-hexagonally packed cavities. In principle one can force a water-front to move along such a surface in an arbitrary direction. Here we focus on just one of the possible directions.

We consider solvent fronts along the $x$-direction, which spread by increasing the volume of the droplet, in the $y$-direction. Recalling that mirror-like boundary conditions are implemented in $x$-, $y$ - and $z$-directions, in this scenario it suffices to have just two rows of cavities, that is $m=2$. The system is much larger in the $y$-direction and we consider $n$ cavities with a spacing $s$. For a hexagonal packing of cavities the distance $s$ and the spacing $t$ between rows are interconnected and we will mention just one of these parameters. The number of cavities that are considered in the $y$-direction is taken sufficiently large so that there are no boundary effects. The surface is thus sufficiently specified by mentioning the cavity diameter $d$, the distance between the cavities $s$ and the cut-off height $h$, or equivalently $c=h / d$. Typically, we will initiate the calculation by means of some initial guess of the SCF. protocol such that a droplet develops with its symmetry plane along the $y=1 / 2$ boundary.

\section{Example 2: Advancing and receding drop fronts on a slightly hydrophilic hexagonally packed inverse opal}

In Figure 8 we give representative examples of planar solvent droplets with their solvent front (on average) along the $x$-direction. The drops sit with their symmetry plane at $y=1 / 2$. The inverse opal is characterised by the cavity diameter $d=31$, the spacing between the cavities in the $y$-direction $s=30$, the cutoff fraction $c=0.80$, and the number of cavities in the $y$-direction, $n=3.5$. The surface is slightly hydrophilic $\left(\chi_{\mathrm{S}}=-0.3\right)$.
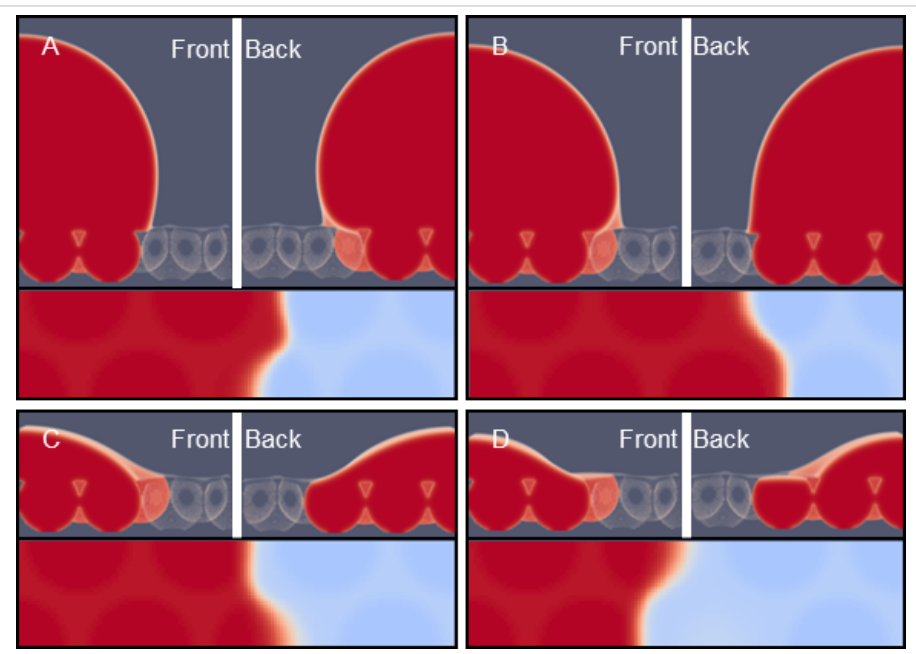

Figure 8: Four examples of droplets on an inverse opal with $d=31, s=30, c=0.80, n=3.5$, and $X_{S}=-0.3$. A,B) Two advancing water fronts with relatively high contact angles; C,D) two receding water fronts with low contact angles. For each panel the left $(z,+y)$, right $(z,-y)$ and bottom images $(x$, $y)$ are for different view-points, that is, the left image is taken from the front, the right image is taken from the back, the bottom image is the top-view. Colour coding: Red is high-density liquid. Blue is low-density gas. White is intermediate density. The snapshots are taken from the calculations of Figure 9. 
The cavities directly under the macroscopic water front are filled with liquid, while the other cavities remain empty. The water front is thus in the Wenzel wetting state (Figure 1) Notice that an additional cavity is filled going from A to B. Such an event gives discontinuities as discussed below. In B there is slightly more liquid in the system than in $\mathrm{A}$, but the height of the drop in A is more than that in $\mathrm{B}$. The liquid in the cavity is noticed as a volume reduction in the drop. The panels $\mathrm{A}$ and $\mathrm{B}$ are taken as examples of the droplet shape in a series of calculations for which the droplet volume was increased (see also below in Figure 9). We refer to these as advancing front lines. The other two panels (C and D) are taken for the situation that the drop volume was decreased and we refer to this situation as receding front lines. Three different view positions of the same drop are given in this figure to illustrate the features that present themselves in advancing and receding cases. From the top-view perspective, we see that the solvent front is not straight. It curves along the cavity openings and the exact shape of the front strongly "fluctuates" depending on the exact value of the droplet volume.

In one of the cases the front is at lower $y$-values at low $x$-values and in the other case it is inversed, the lowest $y$-value is at a high $x$-values. In the four cases shown in Figure 8 we see that the absolute value of differences in the $y$-position does not depend much on the advancing or receding modes.

As the three-phase contact line is curved, necessarily the contact angle must vary as well. The contact angles are best viewed from the side. The top graphs in Figure 8 are images taken from a "front" or "back" view point. We present both of these to illustrate that the shape in the $(z, y)$-plane depends slightly on the $x$-coordinate. Clearly, there is a huge difference in the contact angle between the advancing fronts (very high angles) and the receding fronts (very low angles). Furthermore, as can be seen in panel $\mathrm{D}$, the receding droplet remains pinned on top of the liquid-filled cavity, resulting in a longer contact line as compared to advancing droplets.

For a given snapshot we can evaluate the contact angle $\theta(x, z)$ in the $(z, y)$-plane by estimating by interpolation the position $y^{\prime}$ of the interface, where the $\varphi\left(x, y^{\prime}, z\right)=0.5$. In other words, the $y^{\prime}$-position of the liquid/vapour interface depends both on $x$ and $z: y^{\prime}=y^{\prime}(x, z)$. Then the local contact angle of the solvent front is a function of both $x$ and $z$ :

$$
\theta(x, z)=\arctan \left(y^{\prime}(x, z)-y^{\prime}(x, z-1)\right)
$$

which implies that the contact angle can only be computed for $z>2$. The average angle at a height $z$ is found by averaging along the $x$-direction:

$$
\theta(z)=\frac{1}{M_{x}} \sum_{x=1}^{M_{x}} \theta(x, z)
$$

while the standard deviation $\Delta \theta(z)$ measured in the $x$-direction is given by

$$
\Delta \theta(z)=\sqrt{\frac{1}{M_{x}} \sum_{x=1}^{M_{x}}(\theta(x, z)-\theta(z))^{2}} .
$$

Similarly, the position of the interface $y^{\prime}(x, z)$, the average position of the interface $y^{\prime}(z)$ and the standard deviation $\Delta y^{\prime}(z)$ are straightforwardly recorded.

In Figure 9A we plot the standard deviations $\Delta y^{\prime}(z)$ as a function of $z-h$ (height above the substrate), and in Figure 9B $\theta(z)$
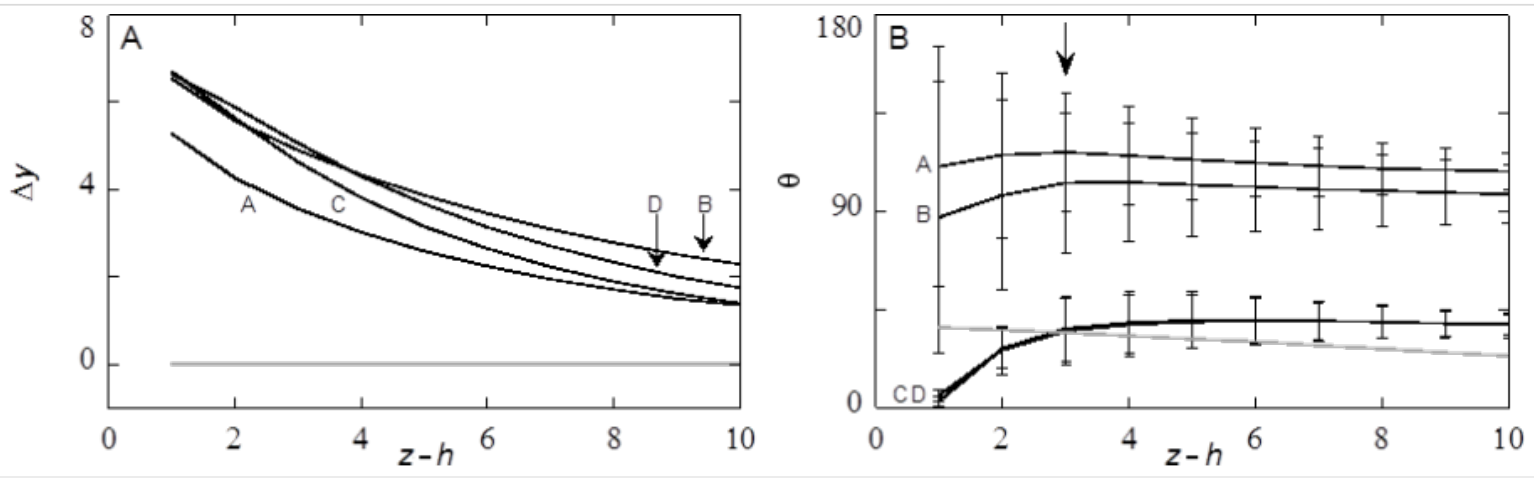

Figure 9: Examples of drop characteristics. A) The standard deviation (measured in $x$-direction) of the position of the interface $\Delta y^{\prime}$ as a function of the height above the substrate $z-h$. The horizontal grey line represents the result on a smooth surface. B) The angle of the liquid/vapour front in the $(z, y)$-plane, $\theta(z)$ together with the standard deviation of the angle measured in the $x$-direction plotted as "error" bars. The grey curve is the contact angle of a similarly sized droplet on a smooth surface. The labels A-D correspond to the snapshots A-D in Figure 8: A,B advancing contact liquid front; C,D are receding liquid fronts. 
together with the fluctuation in the angle $\Delta \theta(z)$ as "error" bars as a function of $z-h$ for the four droplets already shown in Figure 8 is plotted.

It is natural to expect that when the drop characteristics are considered further away from the surface that the influence of the surface is gradually reduced. This is why the $\Delta y^{\prime}(z)$ is a decreasing function of $z$. Again, as noticed already from the snapshots, the value of $\Delta y^{\prime}(z)$ does not depend much on the advancing or receding modes of wetting. That is why the four curves in Figure 9A are nearly the same. We refrain from trying to provide further comments about the differences. Similarly, far from the surface the angles become independent on $x$. That is why in Figure 9B the "error" bars diminish in size when $z-h$ is increased. Indeed very close to the substrate $z-h<4$ the fluctuations are very large, that is about $50 \%$ of the value of $\theta$.

We already noticed that the advancing contact angles are much larger than the receding ones. Figure 9B gives the numerical values more accurately: The advancing angles are on average larger than $90^{\circ}$, whereas the receding angles are about $45^{\circ}$, very close to the angles found for the unstructured surface (grey line). Interestingly, the average contact angle in the advancing mode can go through a small local maximum at a height $z-h=3$. Such an effect hints to the presence of a foot on the droplets. However, at this height the fluctuations are large and we are hesitant not to over-interpret the results.

It is clear that, if we want to compare droplets and see trends, we need to reduce the outcome of the computations. That is why from hereon we will focus on the properties of the droplets on a height of $z-h=3$ (as indicated by the arrow in Figure 9). At this height above the substrate the "foot" is not disturbing too much, while the structure of the surface is still well notice- able. We thus define (if not mentioned otherwise) the fluctuations of the liquid front measured as the standard deviation along the $x$-direction as $\Delta y=\Delta y^{\prime}(h+3)$, and the average contact angle of the drop $\theta=\theta(h+3)$, as well as the fluctuations $\Delta \theta=\Delta \theta(h+3)$.

The calculations of Figure 8 and Figure 9 were started with an initial amount of liquid $\Gamma=3 \cdot 10^{6}$. More liquid is added to the system and these molecules are consumed by the drop. Hence the drop volume increased. Typically we performed ten liquid addition steps and for each of these new profiles are calculated to obtain an advancing angle. The amount of the liquid is subsequently stepwise decreased to check for hysteresis and to obtain a receding angle. The structural properties of the drops are recorded during this cycle and the results were collected in Figure 10.

The starting point of the calculations is not extremely well defined in terms of advancing or receding states. The initial guess takes the system in this case close to an advancing situation: the contact angle $\theta$ is rather high. Typically this initial drop is disregarded from our averaging (see below). Upon stepwise increase of the drop volume (closed spheres) $\Delta y$ decreases from 5.5 to 4.0 while the contact angle $\theta$ increases gradually until point $\mathrm{A}$ is reached. The contact line did not move upon adding the liquid: The contact line is arrested as the contact line cannot be placed on top of a (water filled) cavity [45]. Then with a small increase the system jumps from A to B. Above we saw that in this event one extra cavity is filled with liquid. At this event the contact line de-pins jump-like in an event that may be referred to as a de-pinning transition [19]. In Figure $8 \mathrm{~A}, \mathrm{~B}$ we see that the three-phase contact line has the opposite curvature in the $x$-direction. At this de-pinning event the average contact angle jumps downward to $\theta \approx 90^{\circ}$ (cf.
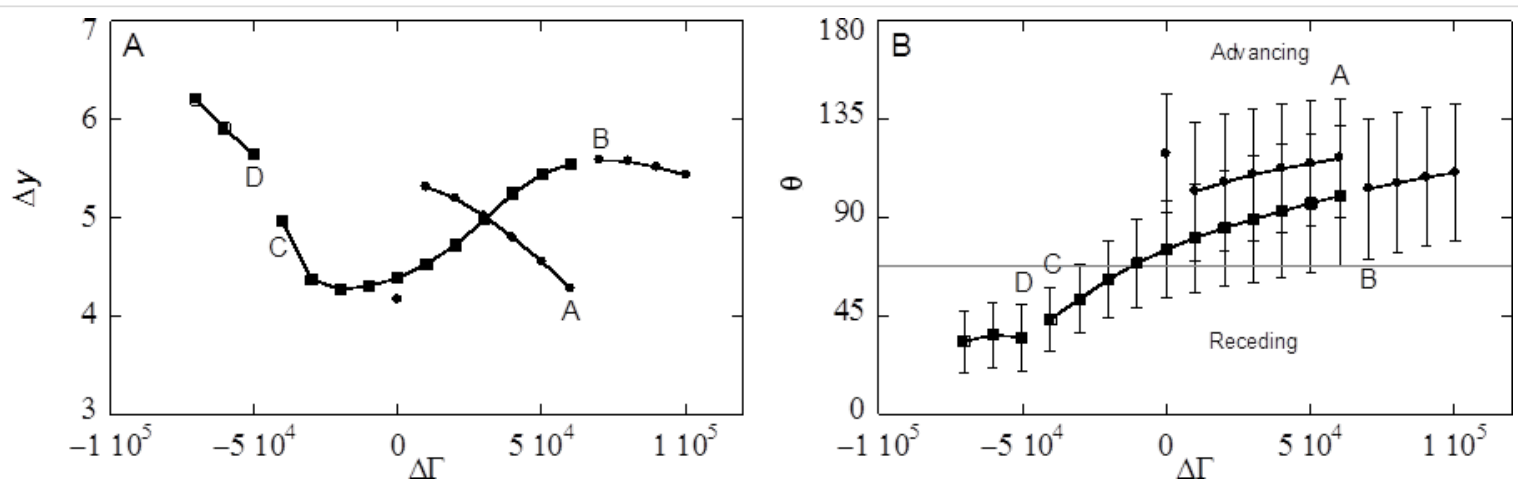

Figure 10: Examples of structural features of advancing and receding drop fronts. A) The fluctuations of the position of the liquid front along the $x$-direction at a height $z=h+3$ as a function of the $\Delta \Gamma$ of the droplet component with an initial amount $\Gamma=3 \cdot 10^{6}$. B) The corresponding average contact angle $\theta$ (measured at a height $z=h+3$ ) and the standard deviation (plotted as error bars). The advancing liquid front is given by the solid sphere data points, the receding ones in solid square data points. The lines are to guide the eye. A discontinuity in the line represents a jump-wise change of the drops on the substrate. The labels A-D correspond to the snapshots of Figure 8 and the structural data of Figure 9 . A and B are along the advancing branch, whereas $C$ and $D$ are taken from the receding drop fronts. Parameters are similar as in Figure 8 and Figure 9. 
Figure 10B) and $\Delta y$ jump-like increases from 4.0 to 5.5 (cf. Figure 10A). The contact angle close to the surface and directly in front of the cavity that fills up, switches thereby from $\theta>90^{\circ}$ to $\theta \approx 90^{\circ}$ (cf. Figure $8 \mathrm{~A}, \mathrm{~B}$ ). These increased contact angles with respect to $\theta_{\mathrm{Y}}\left(68^{\circ}\right.$ for $\left.\chi_{\mathrm{S}}=-0.3\right)$ are hereby found for a hydrophilic surface without air entrapment. The system may experience more of such events when more and more liquid is added. After another four additions of volume we were close to the initial condition and the advancing contact line calculations were stopped.

For receding water fronts (Figure 8C,D), computed by taking liquid out of the system (the squares in Figure 10), we first retrace a part of the advancing curve, that is, the four latest volume additions were undone and the same results were recovered. However, as soon as the volume of the drop is decreased compared to point $\mathrm{B}$ we follow a different route. We do not jump to point $A$, but rather follow the trend downward for the average contact angle $\theta$ (cf. Figure 10B) while also the fluctuations $\Delta y$ decrease. Close to the initial volume the contact angle is found to be close to the value on the smooth surface $\theta=\theta_{Y}$. At this point the curvature of the dependence $\Delta y(\Delta \Gamma)$ changes. Further reduction of the volume of the drop leads to a local minimum of $\Delta y$, while the contact angle drops significantly below the value of $\theta_{\mathrm{Y}}$. Then point $\mathrm{C}$ is reached (cf. Figure $8 \mathrm{C}$ ). Now the receding front is pinned on top of a liquid filled cavity. The low contact angle on top of the cavity, clearly visible for the right-hand site of droplet Figure $8 \mathrm{C}$, is explained by the fact that the liquid wets a surface of the same material (namely the liquid in the cavity). During receding, the water front is thus pinned at the liquid/liquid surface with a local $\theta$ of $0^{\circ}$. The cavity remains filled after the droplet has retracted from the cavity (see cavity on the right-hand site of droplet D) which occurs once again step-wise. During this de-pinning step the three-phase contact line rearranges its curvature again (cf. Figure 8C,D). At this de-pinning transition the value of $\Delta y$ increases jump-like, while the average contact angle decreases somewhat. Upon further reduction of the drop volume the $\Delta y$ increases further while the contact angles remain low.

The traces of Figure 10A,B imply a hysteresis: The curves for adding and reducing volume only overlap when no de-pinning transition has occurred in between the addition or removal steps. The contact line of the advancing droplet experiences a surface consisting of solid and vapour, whereas the contact line of the receding angle experiences a surface consisting of solid and liquid. The true receding angle is only visible for values of $\Delta \Gamma$ between $-5 \cdot 10^{4}$ and $-7 \cdot 10^{4}$ (last three data points) and is about $35^{\circ}$. If the surface can be regarded as consisting of a solid with a liquid, then the contact angle can be calculated using [15]

$$
\cos \theta_{\text {water in cavity }}=1-\Phi_{\mathrm{S}}+\Phi_{\mathrm{S}} \cos \theta_{\mathrm{Y}}
$$

with the fraction of solid on the top of the substrate $\Phi_{\mathrm{S}}=0.43$ (for $c=0.8$ ). The contact angle according to this calculation $\left(40^{\circ}\right)$ is in agreement with the contact angle we find here. For these last droplets, high values of $\Delta y$ are found. The contact line is in these cases pinned on the farther edge of a water-filled cavity (see Figure 8D).

\section{Trends in the shape of advancing water fronts}

In the remainder of this paper we will focus on advancing contact angles. Referring once again to the results of Figure 10B, we typically initiated calculations with the drop near the lower boundary on the $y$-axis in such a way that the resulting drop shape assumes properties of an advancing one. Then, as in Figure 10B ten subsequent increases of the amount of the liquid component were implemented. The average contact angle $\theta$ (along the $x$-direction and at $z=h+3$ ) were again averaged over these ten droplets to obtain $\langle\theta\rangle$. Typically one or more de-pinning events were accepted in this averaging. Recall, that in the advancing branch the contact angle in a de-pinning event changes only slightly. The standard deviations were averaged similarly.

\section{Effect of cavity size $d$}

The size of the inverse opals in the calculations, which is linked to experimental sizes by the width of the interface, invariably is much smaller than the size of inverse opals used in experiments. To study the effect of size of the structures on the (double) averaged advancing contact angle $\langle\theta\rangle$, droplets on inverse opals with cavity diameters ranging from 9 to 52 have been recorded. The trend observed for this series of sizes, can be extrapolated to even bigger sizes without the need to calculate those. In these calculations the spacing between the cavities was set to $d-1$, that is, the cavities were slightly overlapping so that a small hole connects the cavities. The cut-off fraction is kept at $c=0.8$.

As shown in Figure 11, the $\langle\theta\rangle$ is an increasing function of the cavity diameter $d$. For $d>40\langle\theta\rangle$ reaches a plateau. The levelling off hence implies that it is not necessary to increase the cavity sizes even more to reach the experimental limits.

The fact that $\langle\theta\rangle$ can increase above $\theta_{Y}$ is attributed to the pinning of the contact line around the cavities. For very small cavities we observed that the average angle can be smaller than $\theta_{\mathrm{Y}}$ (grey horizontal line in Figure 11). Small cavity sizes are similar to the small confinements $D$ used in the one-gradient slits of Figure 5. From these slit calculations we know that small values of $D$ need only a small oversaturation to fill the slit with the liquid. Similarly, very small cavities can easily be 


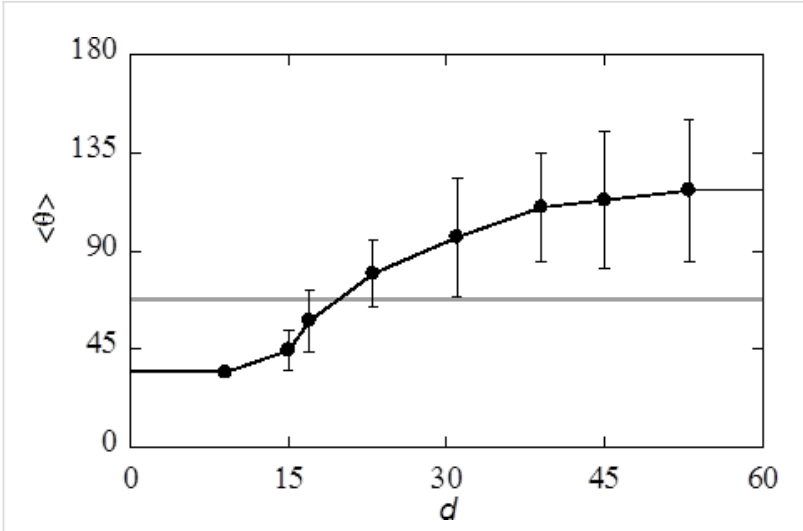

Figure 11: Advancing averaged contact angle $\langle\theta\rangle$ and standard deviation as a function of the cavity diameter $d$ (in lattice units) for a hexagonally ordered inverse opal with cut-off fraction $c=0.8$, distance between cavities $s=d-1$ and slightly hydrophilic surface properties $X_{S}=-0.3$. The grey line is contact angle $\theta_{Y}$ on a smooth surface.

filled with the liquid. We refer to this situation as the impregnated wetting state (Figure 1). Indeed the inverse opal with $d<10$ are in the impregnated state and it is natural to expect that for this case $\langle\theta\rangle<\theta_{\mathrm{Y}}$ : the effective top surface in front of the water front consists of the solid with patches of liquid, just as is the case for a receding droplet. The advancing contact angle $\langle\theta\rangle$ found for these structures (see Figure 11) corresponds to the receding contact angles presented in Figure 10B.

For the structure with $d=15$ the situation is rather complex. It appears that some, but not all, cavities in front of the droplet are filled with liquid. In the process of advancing the liquid front, we add more and more of the liquid. As soon as an additional cavity is filled, the volume for the droplet decreases and this reduces the curvature and the corresponding oversaturation in the vapour phase. This decrease in oversaturation after filling subsequent cavities prevents other cavities to fill up. The number of cavities filled in front of the water front, depends on the size of the droplets on top of the inverse opal: the smaller the droplet, the higher the curvature, thus the more oversaturation. The more cavities are filled, the lower is the advancing contact angle $\langle\theta\rangle$ as the surface becomes effectively hydrophilic. The droplet size dependence will imply small changes in the dependence of $\langle\theta\rangle$ as a function of $d$ in Figure 11. For the drop sizes used to compute Figure $11\langle\theta\rangle=\theta_{\mathrm{Y}}$ occurs approximately at $d=20$.

For the inverse opals with $d \geq 20$ only cavities directly under the droplet are filled (Wenzel wetting state). The water front encounters the same fraction of flat solid top surface, $\Phi_{\mathrm{S}}$ for all cavity sizes, but the increase in $\langle\theta\rangle$ with $d$ depends on the strength of the pinning effects and this allows $\langle\theta\rangle$ to increase with $d$ above $\theta_{\mathrm{Y}}$.

\section{Effect of the $S / L$ interaction parameter XS}

Let us next focus on the interaction of the liquid with the substrate via the $\mathrm{S} / \mathrm{L}$ interaction parameter $\chi_{S}$. The more negative this parameter the more hydrophilic (solvophilic) the surface is. When the $\chi_{S}$ is positive we may refer to the surface as hydrophobic (solvophobic). We select for this study hexagonally ordered inverse opals with a cavity size $d=39$. As shown in Figure 11 such cavity sizes give wetting features in the plateau region where the size dependence was essentially lost. We consider here the case that the cut-off fraction is $c=0.9$. This $c$-value is chosen to mimic the inverse opal in [10]. The distance between the cavities was set to $s=d-1$ (cavities are connected to each other by small openings).

In Figure 12 the grey curve represents the contact angle $\theta_{Y}$ on the smooth surface as a function of $\chi_{\mathrm{S}}$ and this result is reproduced from Figure 5 for ease of comparison. The average advancing contact angle $\langle\theta\rangle$ increases with decreasing hydrophilicity (solvophilicity) of the substrate. However, for $\chi_{\mathrm{S}}>-0.6$ the advancing contact angle is systematically above $\theta_{\mathrm{Y}}$, whereas it is systematically below $\theta_{\mathrm{Y}}$ when $\chi_{\mathrm{S}}<-0.6$.

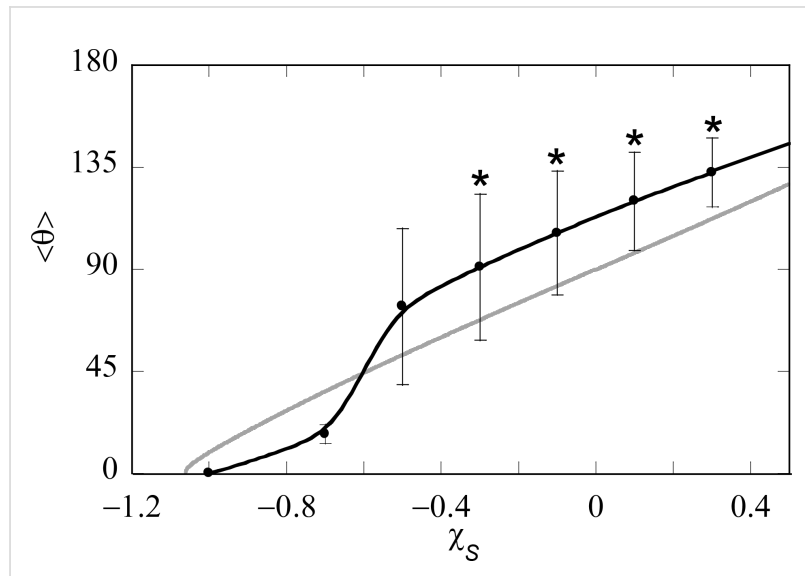

Figure 12: Advancing contact angle $\langle\theta\rangle$ and the corresponding fluctuations (measured along the $x$-direction) indicated as error bars, as a function of the affinity of the solvent for the substrate phase $X_{S}$ for a hexagonal inverse opal with cavity sizes $d=39$, cut-off fraction $c=0.9$ and spacing between the cavities $s=d-1=38$. The contact angle on a smooth surface is shown with a grey line (copied from Figure 4). Stars indicate that the cavities under the water front remain empty (Cassie-Baxter wetting state).

The wetting transition, which is the point for which $\theta$ becomes 0 , occurs at a value of $\chi_{\mathrm{S}}$ which is slightly less hydrophilic for the inverse opal as for the smooth surface. In the low contact angle cases all cavities under the droplets are filled (impregnating wetting state). The filled cavities render the surface slightly more hydrophilic than the smooth surface (see Equation 1) and this caused the early wetting transition for the inverse opal as compared to the smooth surface. 
The inverse opal remains in the impregnating wetting state for small but finite contact angles $\chi_{\mathrm{S}}=-0.7$. In these cases the advancing contact angle is lower than the corresponding $\theta_{\mathrm{Y}}$. The wetting switches to the Wenzel state by increasing the hydrophilicity further to $\chi_{S}=-0.5$. Now the advancing contact angle is larger than $\theta_{Y}$. Eventually, the wetting switches to a Cassie-Baxter wetting state for higher values of $\chi_{\mathrm{S}}$. These cases are labelled by the asterisk in Figure 12. Again the advancing contact angle is larger than $\theta_{Y}$, and the difference $\langle\theta\rangle-\theta_{\mathrm{Y}}$ is roughly constant, that is, it does not depend whether there is the Wenzel or the Cassie-Baxter state. This result suggests that for the advancing angle, it does not matter whether air is entrapped underneath (observed for $\chi_{S} \geq-0.3$ ) the droplet or not (observed for $\chi_{S} \geq-0.5$ ). This implies that the increase in observed $\langle\theta\rangle$ compared to $\theta_{\mathrm{Y}}$ cannot be explained in terms of wetting state. Rather, pinning of the contact line and the de-pinning transition should be considered. The immobilization of the contact line on the kinks in the surface (as explained in Figure 2) on this hexagonally packed inverse opal induces the contact line to curve and extend $(\Delta y>0)$, which is energetically unfavourable. The contact line is even further extended after a de-pinning transition (increase in $\Delta y$ ), indicating that in the stress perpendicular to the surface has increased. However, $\langle\theta\rangle$ decreased locally to values close to $\theta$ on a smooth surface, showing that stress in the vertical direction is released after the transition. The average $\langle\theta\rangle$ is still higher than $\theta_{Y}$ directly after the de-pinning transition. This is caused by the build-up of stress at the other cavity (we consider two rows of cavities).

The interplay between curvature perpendicular to the surface (here discussed in terms of $\langle\theta\rangle$ ) and parallel to the surface (here discussed in terms of $\Delta y$ of the contact line) results in an overall high $\langle\theta\rangle$. Whether the cavity under the moving water front fills with liquid or remains filled with air depends on $\chi_{S}$ : Air is entrapped for more hydrophobic materials, but the cavities fill for a more hydrophilic material. The pinned water front on a surface with higher $\chi_{S}$ can withstand higher $\langle\theta\rangle$ before stress starts to build up and the de-pinning transition occurs.

\section{Effect of cut-off height $c$ at constant spacing $s$}

Experimentally one can control the inverse opal structure by the cut-off height $h$, or equivalently to the cut-off fraction $c$. It is of interest to consider the effect of the cut-off height from a computation point of view. We can study this for a fixed spacing $s$ between the cavities (in this section) or with a fixed fraction of solid in the top of the substrate $\Phi_{\mathrm{S}}$ (next section). In both cases we choose slightly hydrophilic substrates with $\chi_{S}=-0.3$. This value of $\chi_{\mathrm{S}}$ gives $\theta_{\mathrm{Y}}=68^{\circ}$. Similarly as in the previous paragraph we fix $d=39$ and $s=d-1$. Obviously the limits $c=0$ and $c=1$ are the same as the smooth surface. In between these two limits the surface structure is characterised by a top surface layer with a fraction of $\Phi_{\mathrm{S}}$ of the sites being the solid. The top layers are given in two-gradient contour plots in Figure 13C. When $c=0.5$ the amount of $\mathrm{S}$ (dark blue colour) is minimal: both in the limits $c=0$ and $c=1$ the cross-sections are completely blue (not shown).

Correspondingly, the contact line fluctuations, as monitored by $\Delta y$, are averaged over ten "snapshots" while increasing the drop volume. The result is given by $\langle\Delta y\rangle$. In both limits $c=0$ and $c=1$ the surface is ideally smooth and the three-phase contact line will not fluctuate in the $x$-direction. In between these limits $\langle\Delta y\rangle>0$ because the contact line becomes pinned.

The advancing contact angle $\langle\theta\rangle$ together with the standard deviation of this angle (measured in the $x$-direction) is presented in Figure 13A as a function of the parameter $c=h / d$. The corresponding fluctuations of the three-phase contact line $\langle\Delta y\rangle$ (measured in the $x$-direction at a height $z=h+3$ ) are given in Figure 13B.

Inspection of Figure 13A shows that by far the most interesting region is for $0.5<c<1$. For $c<0.5$ the average advancing contact $\langle\theta\rangle$ hardly differs from $\theta_{\mathrm{Y}}$. However, for small values of the cut-off fraction the contact line is already significantly curved (Figure 13B) and the surface structure alters $\theta(x)$ locally to hydrophobic values $\theta>90^{\circ}$. The average $\langle\theta\rangle$ for $0.51<c<0.95$ is higher than $\theta_{\mathrm{Y}}$ on a smooth surface, though locally, $\theta(x)$ may be smaller. The variation of $\theta$ along the $x$-direction (depicted as vertical line) extends to values $\theta<68^{\circ}$ for most values of $c$. A maximum in $\langle\theta\rangle$ is found at $c \approx 0.80$, with $\langle\theta\rangle=110 \pm 30^{\circ}$. A local minimum in $\Delta y$ at this value of $c$ is found, which likely is coupled to the need to keep the overall curvature in the drop constant.

Two parameters of the surface structure that are important for contact line pinning are changed when $c$ is varied: the fraction flat solid top surface $\Phi_{S}$, which is the fraction of flat solid at the top of the surface (dark blue in Figure 13C) and the angle of the kink, $\theta_{\text {kink }}$ (see section S2 of Supporting Information File 1 for calculation of $\theta_{\text {kink }}$ and section S3 of Supporting Information File 1 and Equation 16 for the calculation of $\Phi_{\mathrm{S}}$ ). Both, $\Phi_{\mathrm{S}} \cdot 100$ and $\theta_{\text {kink }}$ are plotted in Figure 13A. The value of $\Phi_{\mathrm{S}} \cdot 100$ equals 100 in both limits of $c$ and has a minimum at $c=0.5$. $\theta_{\text {kink }}$ is $180^{\circ}$ at $c=0$, and decreases to 0 at $c=1$. The latter parameter is considered important for hydrophilic materials to obtain hydrophobic contact angles. It has been suggested that only for $\theta_{\mathrm{Y}}<\theta_{\text {kink }}$ pinning can occur, and a barrier for the water front to enter the cavities is obtained, resulting in air entrapment and the possibility of obtaining higher contact angles $\theta$. However, in our case, $\theta_{\text {kink }}$ is smaller than $\theta_{\mathrm{Y}}$ for most values of $c$, and no jump in $\langle\theta\rangle$ is observed between $c=0.51\left(\theta_{\text {kink }}>\theta_{\mathrm{Y}}\right)$ and 

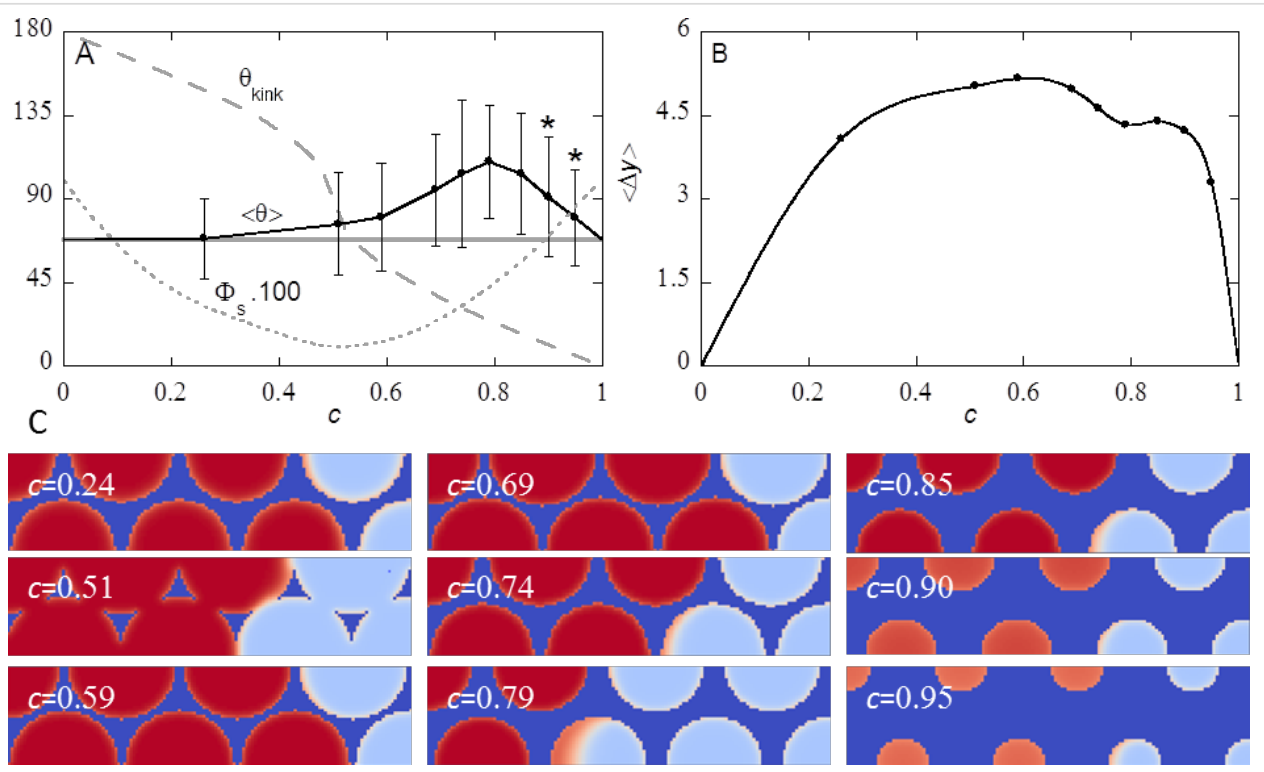

Figure 13: A) Advancing contact angle $\langle\theta\rangle$ and the corresponding standard deviation as measure of variation in the $x$-direction as function of the cutoff fraction $c$. The horizontal grey line is the contact angle $\theta_{Y}$ on a smooth surface, the grey dashed line is the angle of the kink $\theta_{\text {kink }}$ at different values of $c$ and the grey dotted line is $\Phi_{\mathrm{S}} \cdot 100$. Stars indicate that the cavities under the water front are filled with vapour (Cassie-Baxter wetting state). B) Corresponding average variation in $y$-position $\langle\Delta y\rangle$ at $z=h+3$ as function of the cut-off fraction $c$. The hexagonally ordered inverse opal has cavity diameter $d=39$, spacing between the cavities $s=d-1=38$, and $X_{S}=-0.3$. C) The distribution of solid $S$ at the top layer of the substrate, that is $\varphi_{S}(x, y, h)$. The dark blue colour implies density of the solid is unity. The red colour indicates that the opening of the cavity is filled by the solvent. The light blue colour means that the cavity opening is filled with vapour. Orange colour implies that the solvent density is in between the liquid and the vapour. The value of $c$ is indicated.

$c=0.59\left(\theta_{\mathrm{kink}}<\theta_{\mathrm{Y}}\right)$, and no air entrapment is found for $0.59<c<0.85$.

For $c=0.26, \theta_{\text {kink }}$ is too large for pinning to occur according to the argument presented Figure 2. However, an advancing water front is immobilized at the front of a cavity, and moves in one step to a position in front of the next cavity. Hence, despite of the small $\theta_{\text {kink }}$ in our calculations a true de-pinning transition is found.

In our case the pinning of the contact line is due to the complex 3D-structure of the surface: the cavities are placed close to each other, and the contact line thus encounters multiple cavities on a short distance. If the water front was not pinned at the front of a cavity, but rather partly filled the cavity, this would result in a larger L-S interface and a longer contact line. This is apparently energetically more unfavourable than pinning the contact line in front of a cavity. Hence, the contact line pinning is governed by the $3 \mathrm{D}$-structure, and not by the simple $1 \mathrm{D}$-argument presented in Figure 2.

When looking at the top surface (Figure 13C) for $c=0.26$, we see that some cavities on the left are filled (red), while the last 1.5 cavities are not filled (light blue). The last upper cavity is just in front of the contact line. Some liquid (red colour) is present at this value of $z$. Moreover, the situation for $c=0.74$, which has a similar top surface but a different $\theta_{\text {kink }}$, is comparable.

For $c$ close to 0.5 , the substrate is not a continuous structure, but consists of discrete triangular "pillars" (see Figure 13C). Contact line pinning also occurs for discrete shapes $[19,46]$. Assuming that pinning is mainly found on the top surface in line with observations for $c=0.26$, the length over which pinning can take place is limited. However, also in this case a de-pinning transition is observed for an advancing water front. Hence the pinning is not limited to the flat part of the top surface (the dark blue surfaces in Figure 13C) for all values of $c$, but pinning occurs over the $3 \mathrm{D}$ structure.

Decreasing or increasing $c$ from $c=0.5$ results in a continuous top layer. The contact line can hereby be pinned at the front of every cavity. For higher values of $\Phi_{S}$, the contact line is expected to be exclusively located on the top surface (and thus at constant $z$ ). Higher values of $\Phi_{\mathrm{S}}$ (thus smaller cavities) also result in a surface that is more similar to a smooth surface.

In Figure 13C, the liquid-filled cavities are shown in red, whereas vapour-filled cavities are light blue. This is observed for all $c$ up to $c=0.85$. However, for $c=0.90$ and $c=0.95$, we observe a colour in between red and light blue for cavities under the droplet (left hand side). This suggests that at that height, 
neither a liquid, nor a vapour phase is present, and thus that the interface is located at that value of $z$.

\section{Effect of cut-off height $c$ at constant $\Phi_{S}$}

One may argue that the true effect of the cut-off height is seen for cases where $h$ (or equivalently $c$ ) is varied at a fixed amount of $\mathrm{S}$ in the top layer of the substrate, that is for fixed value of $\Phi_{\mathrm{S}}$. To do so, one has to vary the inter-cavity spacing $s$ simultaneously when the cut-off height is changed. Note that the true limits $c=0$ and $c=1$ are hard to reach with fixed $\Phi_{\mathrm{S}}$ as it requires odd distances between the cavities.

The behaviour of the advancing contact angle as well as the contact line fluctuations are recorded for this scenario in Figure 14. Here we have chosen $\Phi_{\mathrm{S}}=0.43$, which corresponds to $c=0.79$ (near the maximum) of Figure 13. Note that all the top surfaces used in Figure 14 are similar to the result shown in Figure $13 \mathrm{C}$ for $c=0.79$.

It is natural to compare results between Figure 13 and Figure 14. For values of $c<0.79$, the average contact angles differ very little. In Figure 14 the average angle appears slightly larger. There is one dramatic difference between Figure 13 and Figure 14. Compare, for example, the top surface for $c=0.51$. While in the case of fixed distance between the cavities $s$ there are individual posts with $\Phi_{\mathrm{S}} \approx 0.1$ (Figure 13C), there is a continuous solid phase in Figure 14 as $\Phi_{\mathrm{S}}=0.43$. Nevertheless, the average contact angles were hardly affected.

At $c>0.79,\langle\theta\rangle$ at constant $\Phi_{\mathrm{S}}$ (Figure 14) keeps growing with the increase in $c$, while at fixed $s$ the contact angles decrease again. The decrease in $\langle\theta\rangle$ for $c>0.79$ in the case of constant $s$ (Figure 13) can thus be attributed to the top surface: Higher $c$ result in smaller cavities and thus in a surface that is more similar to a smooth surface. The increase in $\langle\theta\rangle$ found for increasing $c$ at constant $\Phi_{\mathrm{S}}$ implies that, assuming a constant line tension, a higher $\theta_{\text {kink }}$ for a hexagonally packed surface structure gives rise to a higher barrier for a de-pinning transition to occur and thus higher average contact angles can be maintained. The higher average contact angles are produced with lower and lower fluctuations of the shape of the contact line. Eventually, at the limit of $c=1$ the fluctuations must vanish by definition.

Interestingly, it is found that the cavities under the water front remain empty for $c=0.85$ (and up) at $\Phi_{\mathrm{S}}=0.43$, whereas the cavities were filled for $c=0.85$ at $s=38\left(\Phi_{\mathrm{S}}=0.55\right)$. Bringing the openings of the cavities closer to each other thus prevents water from entering the cavity.

\section{Summary and Outlook}

We have implemented a regular solution lattice model to study the wetting on a structurally complex surface. The interaction between vacancies and liquid is parameterised in a regular solution model by the Flory-Huggins parameter $\chi$. This parameter controls the width of the interface between liquid and vapour. The model allows for a detailed description of the solid phase and the liquid-solid interaction parameter $\chi_{\mathrm{S}}$ is the only parameter to control the hydrophilic/hydrophobic (solphophilic/solvophobic) character of the substrate. We find very complex and interesting wetting states when this model is applied to hexagonally ordered cavities in an inverse opal. It is found that the three-phase contact line is curved and becomes pinned at the cavity openings. Under the droplet the cavities can either be filled with water (impregnated; Wenzel) or filled with the vapour (Cassie-Baxter). No discontinuity in the contact angle $\theta$ is observed for water fronts that are either in the Wenzel state or Cassie-Baxter state, implying that the water front shape is not influenced by air entrapment under the water front, but is rather determined by the surface encountered by the contact line. The pinning of the contact line cannot solely be discussed in terms of the kink that a surface structure makes with respect to the top surface, $\theta_{\text {kink }}$. Also, for $\theta_{\text {kink }}>\theta_{\mathrm{Y}}$, de-pinning transitions are
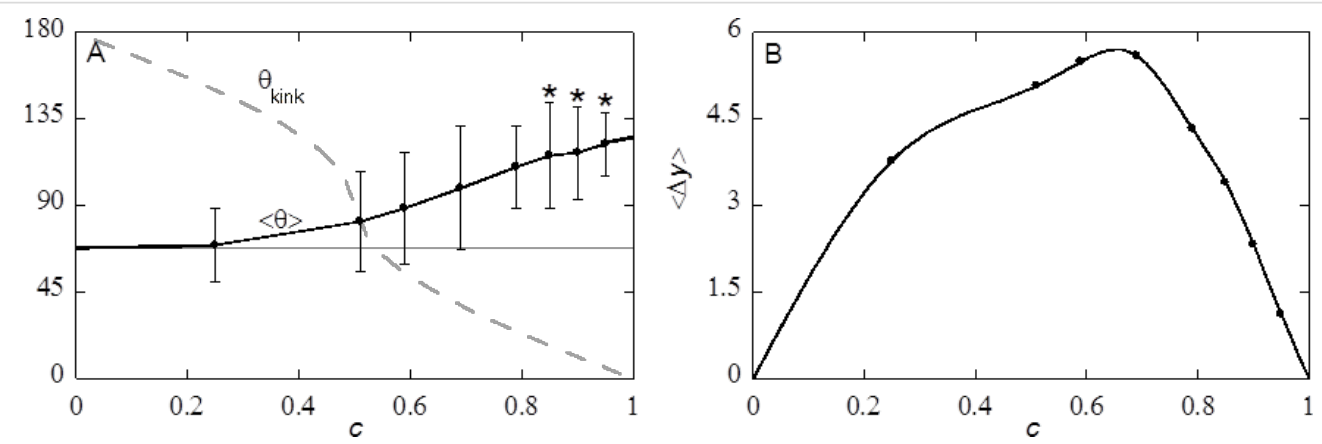

Figure 14: A) The advancing contact angle $\langle\theta\rangle$ and standard deviation as function of cut-off fraction $c$ for fixed value of the solid fraction in the toplayer of the substrate $\Phi_{S}=0.43$. The grey line is $\theta_{Y}$ on a smooth surface and the grey dashed line is the angle of the kink at different $c$. Stars indicate that the cavity under the liquid front are vapour-filled (Cassie-Baxter wetting state). B) Variation in $y$-position $\langle\Delta y\rangle$ as function of cut-off fraction $c$ for $d$ $=39$ and $X_{S}=-0.3$. 
found, and the spacing between the cavities influence whether a cavity under a water front is solvent-filled or vapour-filled. Hence, the full 3D-structure, rather than one parameter $\left(\theta_{\text {kink }}\right)$ should be taken into account. We found a large difference between advancing and receding contact angles, which are also attributed to pinning of the three-phase contact line. More specifically, it was found that while the smooth surface has a contact angle much lower than $90^{\circ}$ the advancing contact angles in the inverse opal can be much larger than $90^{\circ}$. That is, slightly hydrophilic substrates can give hydrophobic contact angles.

Our method can readily be extended to mimic experimental conditions more closely. An interesting case is the wetting of an inverse opal of polypyrrole [10]. The inverse opal of polypyrrole does not have a smooth top surface. Rather, the top surface showed a positive slope radiating from the position of the sacrificial particle. This more complex surface structure can be implemented easily by a more elaborated way to represent the substrate. Furthermore, polypyrrole is a hydrophilic material. The angle $\theta_{\mathrm{Y}}$ on a polypyrrole surface was measured to be about $20^{\circ}$. However, the inverse opal was made using sacrificial polystyrene particles. The particles were removed by dissolving them. This removal was expected to be incomplete, and the contact angle $\theta$ changed to about $80^{\circ}$ for the polypyrrole surface. Polystyrene chains thus must have remained, e.g., in an adsorbed state onto the polypyrrole surface. These adsorbed polymers have a different hydrophilicity, but also changes the local roughness of the surface structures, resulting in both chemical and structural heterogeneities. These heterogeneities on molecular scale can be studied using the approach presented in this paper when the Scheutjens-Fleer machinery is more fully implemented. For example, we can easily consider polymer chains pinned at random locations along the surface of the inverse opal. It is even possible to consider polymer brushes on such substrates which may, e.g., preferentially change wetting characteristics of the top surface or the insides of the cavities [47]. Such decorated substrates may feature dramatic hysteresis effects in the contact angle, because the polymers can stabilize the three-phase contact line while the shape of the cavities induces pinning of the contact line. Hence polymers may introduce a second length scale in inverse opal surface structures.

In the current work we have solved the regular solution model using the self-consistent field (SCF) machinery. Only low memory costs were required to find SCF solutions that are linearly proportional to the volume, that is, the number of lattice sites in the system $\left(M_{x}, M_{y}, M_{z}\right)$. Also the CPU time scales only linearly with the volume. Here we focused on very small systems and used a desktop PC to find accurate results in a few minutes CPU time. Alternatively, the complete set of equations can be solved on a GPU and using CUDA technology the results may be generated 10 to 100 times faster [48]. As a result systems which are 10 times larger in each direction should still be feasible, while keeping the wall-time for the computations at less than an hour. In this case the regular solution model captures the macroscopically relevant sizes and we do not need to rely on extrapolations.

\section{Conclusion}

Regular solution theory is used to study the wetting behaviour of a simplistic molecular model on a complex inverse opal surface topology. The model features molecular input parameters and gives interfacial energies, contact angles and three-phase contact line shapes. As a result, advancing as well as receding wetting front scenarios were considered. It was found that there is a large contact angle hysteresis in these systems, which was attributed to contact line pinning. We have seen that the cavities can be filled by the liquid or remain dry, i.e., filled by vapour. Cavities in front of the droplet may be filled by a capillary condensation effect, while receding contact angles typically do not empty the liquid filled cavities. Interestingly, when the substrate is slightly hydrophilic it is possible that advancing contact angles have contact angles larger than $90^{\circ}$.

\section{Supporting Information}

\section{Supporting Information File 1}

Detailed mathematical calculations.

[http://www.beilstein-journals.org/bjnano/content/ supplementary/2190-4286-7-129-S1.pdf]

\section{Acknowledgements}

S. A. and M. K. acknowledge The Netherlands Organization for Scientific Research (NWO) for financial support.

\section{References}

1. Cheng, Y.-T.; Rodak, D. E. Appl. Phys. Lett. 2005, 86, 144101. doi:10.1063/1.1895487

2. Guo, C.; Feng, L.; Zhai, J.; Wang, G.; Song, Y.; Jiang, L.; Zhu, D. ChemPhysChem 2004, 5, 750-753. doi:10.1002/cphc.200400013

3. Abdelsalam, M. E.; Bartlett, P. N.; Kelf, T.; Baumberg, J. Langmuir 2005, 21, 1753-1757. doi:10.1021/la047468q

4. Zhu, M.; Zuo, W.; Yu, H.; Yang, W.; Chen, Y. J. Mater. Sci. 2006, 41, 3793-3797. doi:10.1007/s10853-005-5910-z

5. Cao, L.; Hu, H.-H.; Gao, D. Langmuir 2007, 23, 4310-4314. doi:10.1021/la063572r

6. Ma, Y.; Cao, X.; Feng, X.; Ma, Y.; Zou, H. Polymer 2007, 48 7455-7460. doi:10.1016/j.polymer.2007.10.038

7. Feng, L.; Song, Y.; Zhai, J.; Liu, B.; Xu, J.; Jiang, L.; Zhu, D. Angew. Chem. 2003, 115, 824-826. doi:10.1002/ange.200390181

8. Hosono, E.; Fujihara, S.; Honma, I.; Zhou, H. J. Am. Chem. Soc. 2005, 127, 13458-13459. doi:10.1021/ja053745j 
9. Karlsson, M.; Forsberg, P.; Nikolajeff, F. Langmuir 2010, 26, 889-893. doi:10.1021/la902361c

10. Akerboom, S.; Pujari, S. P.; Turak, A.; Kamperman, M. ACS Appl. Mater. Interfaces 2015, 7, 16507-16517. doi:10.1021/acsami.5b03903

11. Wenzel, R. N. Ind. Eng. Chem. 1936, 28, 988-994. doi:10.1021/ie50320a024

12. Liu, J.-L.; Feng, X.-Q.; Wang, G.; Yu, S.-W. J. Phys.: Condens. Matter 2007, 19, 356002. doi:10.1088/0953-8984/19/35/356002

13. Marmur, A. Langmuir 2008, 24, 7573-7579. doi:10.1021/la800304r

14. Choi, H.-J.; Choo, S.; Shin, J.-H.; Kim, K.-I.; Lee, H. J. Phys. Chem. C 2013, 117, 24354-24359. doi:10.1021/jp4070399

15. Bormashenko, E.; Bormashenko, Y.; Whyman, G.; Pogreb, R.; Stanevsky, O. J. Colloid Interface Sci. 2006, 302, 308-311. doi:10.1016/j.jcis.2006.06.016

16. Cassie, A. B. D.; Baxter, S. Trans. Faraday Soc. 1944, 40, 546-551. doi:10.1039/tf9444000546

17. Herminghaus, S.; Brinkmann, M.; Seemann, R. Annu. Rev. Mater. Res. 2008, 38, 101-121. doi:10.1146/annurev.matsci.38.060407.130335

18. Papadopoulos, P.; Deng, X.; Mammen, L.; Drotlef, D.-M.; Battagliarin, G.; Li, C.; Müllen, K.; Landfester, K.; del Campo, A.; Butt, H.-J. Langmuir 2012, 28, 8392-8398. doi:10.1021/la300379u

19. Forsberg, P. S. H.; Priest, C.; Brinkmann, M.; Sedev, R.; Ralston, J. Langmuir 2010, 26, 860-865. doi:10.1021/la902296d

20. Bormashenko, E.; Musin, A.; Whyman, G.; Zinigrad, M. Langmuir 2012 28, 3460-3464. doi:10.1021/la204424n

21. Tuteja, A.; Choi, W.; Ma, M.; Mabry, J. M.; Mazzella, S. A.; Rutledge, G. C.; McKinley, G. H. Science 2007, 318, 1618-1622. doi:10.1126/science.1148326

22. Hensel, R.; Helbig, R.; Aland, S.; Braun, H.-G.; Voigt, A.; Neinhuis, C.; Werner, C. Langmuir 2013, 29, 1100-1112. doi:10.1021/la304179b

23. Savoy, E. S.; Escobedo, F. A. Langmuir 2012, 28, 16080-16090. doi:10.1021/la303407r

24. Mugele, F.; Becker, T.; Nikopoulos, R.; Kohonen, M.; Herminghaus, S. J. Adhes. Sci. Technol. 2002, 16, 951-964. doi:10.1163/156856102760136490

25. Wu, J.; Zhang, M.; Wang, X.; Li, S.; Wen, W. Langmuir 2011, 27 , 5705-5708. doi:10.1021/la200697k

26. de Gennes, P. G. Rev. Mod. Phys. 1985, 57, 827-863. doi:10.1103/RevModPhys.57.827

27. Chamakos, N. T.; Kavousanakis, M. E.; Papathanasiou, A. G. Soft Matter 2013, 9, 9624-9632. doi:10.1039/c3sm51377g

28. Patankar, N. A. J. Adhes. Sci. Technol. 2009, 23, 413-433. doi:10.1163/156856108X370073

29. Pashos, G.; Kokkoris, G.; Boudouvis, A. G. J. Comput. Phys. 2015, 283, 258-270. doi:10.1016/j.jcp.2014.11.045

30. Park, J.-Y.; Ha, M.-Y.; Choi, H.-J.; Hong, S.-D.; Yoon, H.-S. J. Mech. Sci. Technol. 2011, 25, 323-332. doi:10.1007/s12206-010-1218-2

31. Kumar, V.; Sridhar, S.; Errington, J. R. J. Chem. Phys. 2011, 135, 184702. doi:10.1063/1.3655817

32. Zhang, Z.; Kim, H.; Ha, M. Y.; Jang, J. Phys. Chem. Chem. Phys. 2014, 16, 5613-5621. doi:10.1039/c3cp54976c

33. Dupuis, A.; Yeomans, J. M. Langmuir 2005, 21, 2624-2629. doi:10.1021/la047348i

34. Pooley, C. M.; Kusumaatmaja, H.; Yeomans, J. M. Phys. Rev. E 2008, 78, 056709. doi:10.1103/PhysRevE.78.056709

35. Vrancken, R. J.; Kusumaatmaja, H.; Hermans, K.; Prenen, A. M.; Pierre-Louis, O.; Bastiaansen, C. W. M.; Broer, D. J. Langmuir 2010, 26, 3335-3341. doi:10.1021/la903091s
36. Kavousanakis, M. E.; Colosqui, C. E.; Kevrekidis, I. G.; Papathanasiou, A. G. Soft Matter 2012, 8, 7928-7936. doi:10.1039/c2sm25377a

37. Blow, M. L.; Kusumaatmaja, H.; Yeomans, J. M. J. Phys.: Condens. Matter 2009, 21, 464125. doi:10.1088/0953-8984/21/46/464125

38. De Coninck, J.; Blake, T. D. Annu. Rev. Mater. Res. 2008, 38, 1-22. doi:10.1146/annurev.matsci.38.060407.130339

39. van der Waals, J. D. Over de Continuiteit van den Gas-en Vloeistoftoestand; AW Sijthoff: Netherlands, 1973.

40. Safran, S. A. Statistical thermodynamics of surfaces, interfaces, and membranes; Addison-Wesley: Boston, MA, U.S.A., 1994.

41. Stiopkin, I. V.; Weeraman, C.; Pieniazek, P. A.; Shalhout, F. Y.; Skinner, J. L.; Benderskii, A. V. Nature 2011, 474, 192-195. doi:10.1038/nature10173

42. Lee, Y.-J.; Braun, P. V. Adv. Mater. 2003, 15, 563-566. doi:10.1002/adma.200304588

43. Li, H.; Vienneau, G.; Jones, M.; Subramanian, B.; Robichaud, J.; Djaoued, Y. J. Mater. Chem. C 2014, 2, 7804-7810. doi:10.1039/C4TC01401D

44. Couturier, J.-P.; Sütterlin, M.; Laschewsky, A.; Hettrich, C.; Wischerhoff, E. Angew. Chem., Int. Ed. 2015, 54, 6641-6644. doi:10.1002/anie.201500674

45. Bormashenko, E. Colloids Surf., A 2008, 324, 47-50. doi:10.1016/j.colsurfa.2008.03.025

46. Gao, L.; McCarthy, T. J. Langmuir 2006, 22, 2966-2967. doi:10.1021/la0532149

47. de Vos, W. M.; Leermakers, F. A. M. Polymer 2009, 50, 305-316. doi:10.1016/j.polymer.2008.10.025

48. Harish, P.; Narayanan, P. J. Accelerating Large Graph Algorithms on the GPU Using CUDA. In High performance computing - HiPC 2007, 14th International Conference, Goa, India, Dec 18-21, 2007; Aluru, S.; Parashar, M.; Badrinath, R.; Prasanna, V. K., Eds.; Springer: Berlin, Germany, 2007; pp 197-208. doi:10.1007/978-3-540-77220-0_21

\section{License and Terms}

This is an Open Access article under the terms of the Creative Commons Attribution License (http://creativecommons.org/licenses/by/4.0), which permits unrestricted use, distribution, and reproduction in any medium, provided the original work is properly cited.

The license is subject to the Beilstein Journal of Nanotechnology terms and conditions: (http://www.beilstein-journals.org/bjnano)

The definitive version of this article is the electronic one which can be found at: $\underline{\text { doi:10.3762/bjnano.7.129 }}$ 